\title{
Expressing 2-keto acid pathway enzymes significantly increases photosynthetic isobutanol production
}

\author{
Hao Xie and Peter Lindblad* (D)
}

\begin{abstract}
Background: Cyanobacteria, photosynthetic microorganisms, are promising green cell factories for chemical production, including biofuels. Isobutanol, a four-carbon alcohol, is considered as a superior candidate as a biofuel for its high energy density with suitable chemical and physical characteristics. The unicellular cyanobacterium Synechocystis PCC 6803 has been successfully engineered for photosynthetic isobutanol production from $\mathrm{CO}_{2}$ and solar energy in a direct process.

Results: Heterologous expression of a-ketoisovalerate decarboxylase (Kivd ${ }^{\text {S286T }}$ ) is sufficient for isobutanol synthesis via the 2-keto acid pathway in Synechocystis. With additional expression of acetolactate synthase (AlsS), acetohydroxyacid isomeroreductase (IIVC), dihydroxy-acid dehydratase (IIvD), and alcohol dehydrogenase (SIr1192 ${ }^{\mathrm{OP}}$ ), the Synechocystis strain $\mathrm{HX} 42$, with a functional 2-keto acid pathway, showed enhanced isobutanol production reaching $98 \mathrm{mg} \mathrm{L}^{-1}$ in short-term screening experiments. Through modulating kivd ${ }^{5286 T}$ copy numbers as well as the composition of the $5^{\prime}$-region, a final Synechocystis strain HX47 with three copies of kivd ${ }^{\text {S286T }}$ showed a significantly improved isobutanol production of $144 \mathrm{mg} \mathrm{L}^{-1}$, an 177\% increase compared to the previously reported best producing strain under identical conditions.
\end{abstract}

Conclusions: This work demonstrates the feasibility to express heterologous genes with a combination of selfreplicating plasmid-based system and genome-based system in Synechocystis cells. Obtained isobutanol-producing Synechocystis strains form the base for further investigation of continuous, long-term-photosynthetic isobutanol production from solar energy and carbon dioxide.

Keywords: Synechocystis PCC 6803, Isobutanol production, 2-Keto acid pathway, a-Ketoisovalerate decarboxylase, Metabolic engineering

\section{Introduction}

As an effective approach to alleviate the increased demand of energy and the concerns of global climate change caused by $\mathrm{CO}_{2}$ emissions, there is a great urgency

\section{Article corrected in 2022}

*Correspondence: peter.lindblad@kemi.uu.se

Microbial Chemistry, Department of Chemistry-Ångström Laboratory,

Uppsala University, Box 523, 75120 Uppsala, Sweden to develop biofuels as new energy carriers to replace presently used fossil resources [1]. Isobutanol, a four-carbon alcohol, is one of the preferred candidates as biofuels due to its superior characteristics, such as high combustion power, low hygroscopicity, and low water solubility [2]. Isobutanol has a similar energy density as 1-butanol but a higher octane number than 1-butanol, (research, motor and pump octane numbers of 114, 94 and 104 compared to 96,78 and 87 , respectively), making it preferred for blending into gasoline and more readily upgraded to renewable jet fuel blendstock [3]. Additionally, isobutanol 
is an important bulk chemical with many direct and indirect applications, including but not limited to be used in plastics, coating, and pharmaceutical industries [3-5].

Biological isobutanol production was first demonstrated in Escherichia coli through the implementation of a non-fermentative pathway, the so-called 2-keto acid pathway [2]. The 2-keto acid pathway, using pyruvate as starting metabolite, involves five enzymes: acetolactate synthase (AlsS), acetohydroxy-acid isomeroreductase (IlvC), dihydroxy-acid dehydrase (IlvD), $\alpha$-ketoisovalerate decarboxylase (Kivd), and alcohol dehydrogenase (Adh) (Fig. 1). As a key enzyme of the 2-keto acid pathway, Kivd decarboxylates 2-ketoisovalerate, an intermediate of the valine synthesis pathway, into isobutyraldehyde, which is further converted to isobutanol by Adh. Later on, the same strategy was extensively applied in various microorganisms, including Saccharomyces cerevisiae [6],

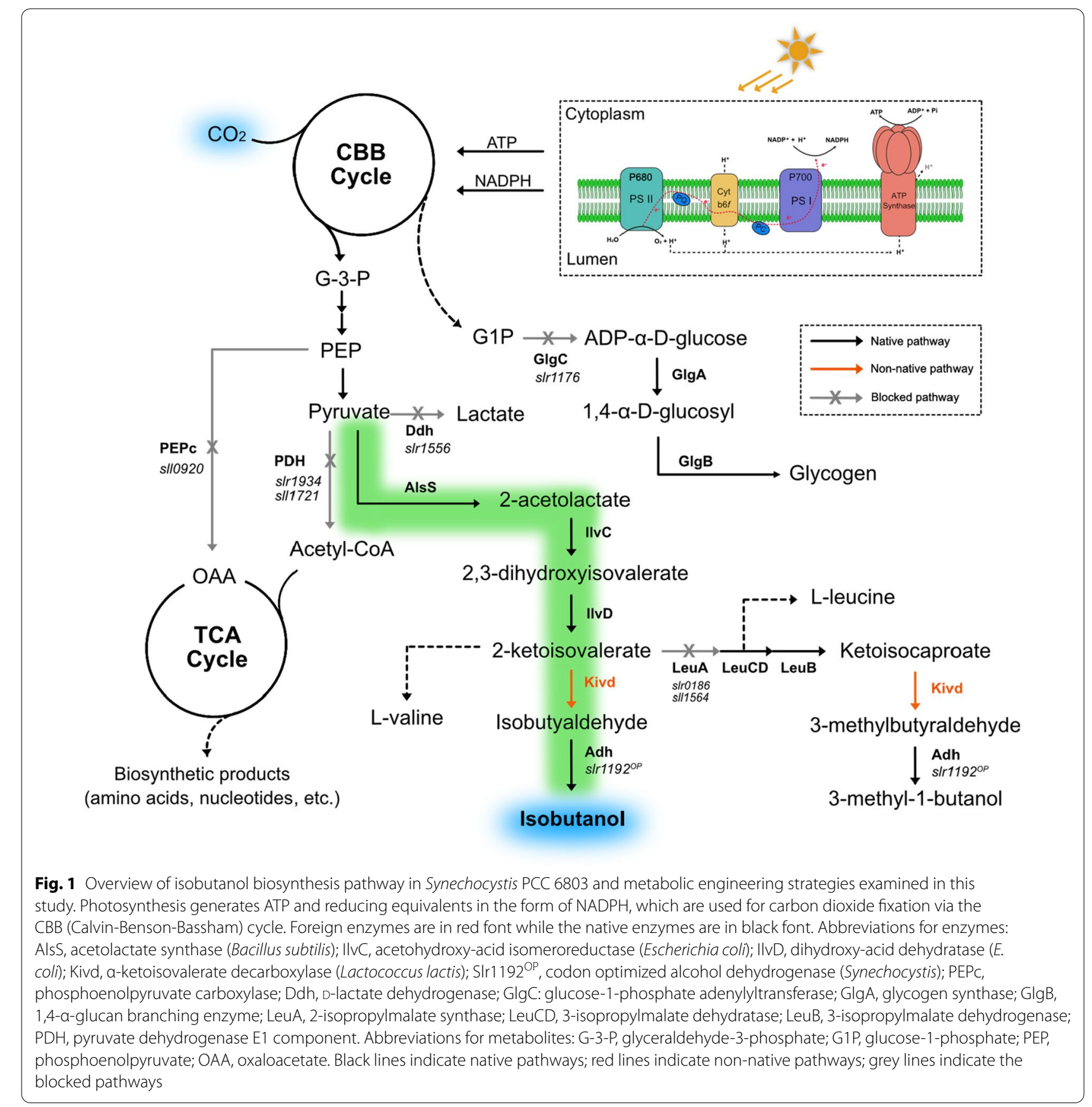


Corynebacterium glutamicum [7], Ralstonia eutropha [8], Clostridium cellulolyticum [9], Bacillus subtilis [10] as well as cyanobacteria [11-14].

Different from heterotrophic microorganisms, cyanobacteria are photoautotrophic microorganisms capable of performing photosynthesis using water and carbon dioxide as substrates and sunlight as energy source. Synechococcus elongatus PCC 7942 (hereafter Synechococcus) was the first cyanobacterium being engineered for isobutanol synthesis, by expressing AlsS from B. subtilis, IlvC and IlvD from E. coli, Kivd from Lactococcus lactis, and Adh (YqhD) from E. coli [11]. In the same study, increased isobutanol production was observed after overexpressing ribulose 1,5-bisphosphate carboxylase/oxygenase (Rubisco), a rate limiting enzyme in the Calvin Benson Bassham (CBB) cycle [11]. Following the first proof-ofconcept study to develop cyanobacteria as biocatalysts to produce isobutanol directly from solar energy and $\mathrm{CO}_{2}$, Synechocystis PCC 6803 (hereafter Synechocystis), another model cyanobacterium, was demonstrated to have the capability for isobutanol synthesis with a single heterologous expression of Kivd from L. lactis, with 3-methyl-1-butanol (3M1B) produced as minor by-product [14]. Moreover, Kivd was identified as one bottleneck in the isobutanol synthesis pathway [14]. In a following study, an engineered $\mathrm{Kivd}^{\mathrm{S} 286 \mathrm{~T}}$ with a single amino acid replacement resulted in higher isobutanol production as well as higher isobutanol-to-3M1B molar ratio than the original Kivd [15]. Apart from genomic modifications, cultivation condition optimization also plays a significant role in further enhancement of isobutanol production in Synechocystis. By stabilizing the $\mathrm{pH}$ to between 7 and 8 using $\mathrm{HCl}$ titration, an increase of isobutanol production of $273 \%$ was observed after 10-day cultivation [12].

As established previously [12], two distinct cultivation methods, a short-term screening experiment and a long-term milking experiment, have been employed for examining isobutanol production in Synechocystis. The short-term screening experiment is used to compare different engineered Synechocystis strains and aims to identify a strain with the best performance of isobutanol synthesis, while the main purpose of a long-term milking experiment is to explore the capacity of isobutanol production under a given environmental condition of a selected Synechocystis strain.

In the present study, short-term screening experiments were used to examine isobutanol production in engineered Synechocystis strains. Our previous best isobutanol producing strain pEEK2-ST, with Kivd $^{\text {S286T }}$ expressed from a self-replicating plasmid, producing $52 \mathrm{mg}$ isobutanol $\mathrm{L}^{-1}$ in short-term screening experiments [12], was used as parental strain. Different strategies were explored to further enhance isobutanol production of Synechocystis cells. Two protein expression systems, a self-replicating plasmid-based system (selected gene placed on self-replicating plasmid) and a genome-based system (selected gene integrated into the chromosome), were introduced simultaneously into the Synechocystis cells. Further optimization of the 2-keto acid pathway was performed by overexpressing selected enzymes from the valine synthesis pathway, with the aim to increase the carbon flux towards the 2-keto acid pathway. Similar to what has been observed in Synechococcus [11], for the first time, an engineered Synechocystis cell with a complete 2-keto acid pathway was successfully generated, with a simultaneous increase in isobutanol production. On the other hand, both transcription and translation of $k i v d^{S 286 T}$ were tuned for enhanced protein expression levels, further contributing to improvement of isobutanol production in Synechocystis cells. Moreover, gene dosage of $k i v d^{S 286 T}$ was manipulated through multiple integrations into different sites of the chromosome and on a self-replicating plasmid, which is confirmed to be positively correlated to isobutanol production of Synechocystis cells.

\section{Materials and methods}

Strains used in cloning, transformation and conjugation

Escherichia coli strains DH5 $\alpha-Z 1$ (Invitrogen) and T7 Express (NEB) were used for cloning. The cells were grown at $37{ }^{\circ} \mathrm{C}$ in lysogeny broth (LB) medium (SigmaAldrich) supplemented with appropriate antibiotic(s). The final concentration used for kanamycin (Thermo Fisher Scientific), chloramphenicol (Sigma-Aldrich) and spectinomycin (AppliChem) were $50 \mu \mathrm{g} \mathrm{mL} \mathrm{m}^{-1}$, $35 \mu \mathrm{g} \mathrm{mL}{ }^{-1}$, and $50 \mu \mathrm{g} \mathrm{mL}^{-1}$, respectively. E. coli $\mathrm{HB} 101$ helper cell with plasmid $p R L 443-A m p^{R}$ was used for conjugation. The glucose-tolerant Synechocystis PCC 6803 strain was used throughout this study. Synechocystis seed cultures were maintained under $30 \mu \mathrm{mol}$ photons $\mathrm{m}^{-2} \mathrm{~s}^{-1}$ at $30^{\circ} \mathrm{C}$ in BG11 medium [16].

\section{Plasmid construction}

All heterologous genes and endogenous genes were codon optimized and synthesized by GenScript and are listed in Additional file 1: Table S1. Homologous recombination regions were amplified using specific primers from wild-type Synechocystis genome using Phusion Polymerase (Thermo Scientific). PpsbA2, RBS*, Terminator BBa_B0015 were amplified from pEERM1 [17]. PtrcBCD and PtrcRiboJ promotor fragments were amplified from pEEC series of vectors [18]. All integrative vectors were based on the recently reported PEERM series of vectors [17]. The homologous recombination regions are the around $1000 \mathrm{bp}$ upstream sequence and the around $1000 \mathrm{bp}$ downstream sequence of the integrated sites in 
the chromosome. BglII, EcoRI, XbaI, SpeI, PstI, BamHI and SalI restriction enzymes were used to assemble all plasmids in this study. All enzymatic digestions were performed with FastDigest enzymes (Thermo Scientific). Ligation reactions were performed using the Quick Ligation kit from New England Biolabs (NEB). The plasmids constructed in this study are listed in Additional file 1: Table S2. The Primers used for plasmids construction in this study are listed in Additional file 1: Table S3.

\section{Transformation methods for Synechocystis Natural transformation}

Synechocystis cells were grown to mid-log phase $\left(\mathrm{OD}_{750}=0.5-1.2\right)$ in liquid BG11 medium. Then cells were collected by centrifuging at $7200 \mathrm{rpm} / 5000 \times g$ for 5 min and washed twice with fresh liquid BG11 medium without antibiotics, and finally resuspended in fresh BG11 medium at a density of $1 \times 10^{9}$ cells $\mathrm{mL}^{-1}$. A total of $400 \mu \mathrm{L}$ of concentrated Synechocystis cells were mixed with $4 \mu \mathrm{g}$ of plasmid DNA. After incubation under illumination of $50 \mu \mathrm{mol}$ photons $\mathrm{m}^{-2} \mathrm{~s}^{-1}$ at $30^{\circ} \mathrm{C}$ for $4-5 \mathrm{~h}$, the cells were spread onto nitrocellulose membranes on BG11 agar plates without antibiotics for another $24 \mathrm{~h}$ incubation. For colony selection and maintenance, the membranes were transferred onto new BG11 agar plates with appropriate antibiotic(s), and incubated under the same conditions as before. Isolated single colony was streaked onto new BG11 agar plates with appropriate antibiotics, and were analyzed by colony PCR using gene specific primers (Additional file 1: Table S3) and DreamTaq DNA polymerase (Thermo Scientific). The verified transformants with expression cassette integrated into the genome were then inoculated into 6-well plate (SARSTEDT) in BG11 medium with corresponding antibiotic(s), and propagated until fully segregation. The segregation of each strain was examined by PCR using gene specific primers (Additional file 1: Table S3) and DreamTaq DNA polymerase (Thermo Scientific) on the extracted genomic DNA, which is prepared based on methods described in [19].

\section{Conjugation}

Synechocystis cells were grown to mid-log phase $\left(\mathrm{OD}_{750}=0.5-1.2\right)$ in liquid BG11 medium. E. coli HB101 conjugative cells with the plasmid pRL443-Amp ${ }^{R}$ and E. coli cells with cargo plasmid were grown overnight at $37{ }^{\circ} \mathrm{C}$. One milliliter of E. coli and Synechocystis cells were centrifuged $(7200 \mathrm{rpm} / 5000 \times g$ for $10 \mathrm{~min})$ at room temperature. The $E$. coli cargo and conjugative cells were resuspended in $500 \mu \mathrm{L}$ of LB without antibiotics, combined with each other and washed once with fresh medium before being resuspended in $500 \mu \mathrm{L}$ of LB without antibiotics. The Synechocystis cells was washed twice with fresh medium and then resuspended in 500 $\mu \mathrm{L}$ of fresh BG11 without antibiotics. A mixture of cargo cells $(250 \mu \mathrm{L})$, conjugative cells $(250 \mu \mathrm{L})$ and Synechocystis cells $(100 \mu \mathrm{L})$ was incubated under illumination of $50 \mu \mathrm{mol}$ photons $\mathrm{m}^{-2} \mathrm{~s}^{-1}$ at $30{ }^{\circ} \mathrm{C}$ for $1.5 \mathrm{~h}$, before being spread onto membranes on BG11 agar plates without antibiotics for $24 \mathrm{~h}$. For colony selection and maintenance, the membranes were transferred onto new BG11 agar plates with appropriate antibiotic(s), and incubated under the same conditions as before. Positive colonies were verified by colony PCR using gene specific primers (Additional file 1: Table S3) and DreamTaq DNA polymerase (Thermo Scientific). All engineered Synechocystis strains generated in this study are summarized in Table 1.

\section{Cultivation condition of short-term screening experiment}

Seed cultures were grown under $30 \mu \mathrm{mol}$ photons $\mathrm{m}^{-2} \mathrm{~s}^{-1}$ at $30{ }^{\circ} \mathrm{C}$ in BG11 with appropriate antibiotic(s) in $100 \mathrm{~mL}$ Erlenmeyer flasks (VWR) until $\mathrm{OD}_{750}=1.5-$ 2.0. The seed cultures were then used to inoculate $25 \mathrm{~mL}$ experimental cultures to $\mathrm{OD}_{750}=0.1$ in BioLite $25 \mathrm{~cm}^{2}$ plug-sealed tissue culture flasks (Thermo Fisher Scientific). The medium used for experimental culture was BG11 with addition of $50 \mathrm{mM} \mathrm{NaHCO}$ (Sigma-Aldrich) and appropriate antibiotic(s) (final concentration: chloramphenicol $20 \mu \mathrm{g} \mathrm{mL}{ }^{-1}$, spectinomycin $50 \mu \mathrm{g} \mathrm{mL}-1$, and kanamycin $50 \mu \mathrm{g} \mathrm{mL}^{-1}$ ). All experimental cultures were prepared in triplicate. The flasks were shaken horizontally at $120 \mathrm{rpm}$, under $50 \mu \mathrm{mol}$ photons $\mathrm{m}^{-2} \mathrm{~s}^{-1}$ at $30{ }^{\circ} \mathrm{C}$. Two milliliter of culture was sampled from each flask every second day for measurements and $2 \mathrm{~mL}$ of fresh BG11 medium with addition of $500 \mathrm{mM} \mathrm{NaHCO}$ (Sigma-Aldrich) and appropriate antibiotic(s) were added back. The cultivation was terminated when the isobutanol production in the culture started to decrease.

\section{RNA isolation and semi-quantitative reverse transcript PCR (RT-PCR)}

Synechocystis cells were harvested at $\mathrm{OD}_{750}=0.3-0.7$. Total RNA was isolated from cell culture using RTI Reagent (Sigma-Aldrich) according to the manufacturer's instructions. The RNA concentration was measured using a Nanodrop ${ }^{\mathrm{TM}} 2000$ spectrophotometer (Thermo Fisher Scientific). $1 \mu \mathrm{g}$ of RNA and qScript ${ }^{\mathrm{TM}}$ cDNA Synthesis Kit were used for cDNA synthesis. 23S RNA was used as control.

\section{Crude protein extraction and SDS-PAGE/ Western-immunoblot}

Proteins were extracted from cell cultures with $\mathrm{OD}_{750}=2.0$, and $5 \mathrm{~mL}$ of cell culture was harvested by centrifugation at $5000 \mathrm{rpm} / 4500 \times g$ for $10 \mathrm{~min}$ at room temperature. Obtained cell pellets were washed with 
Table 1 Synechocystis PCC 6803 strains used in this study

\begin{tabular}{|c|c|c|}
\hline Strain & Relevant genotypes $^{a}$ & Reference \\
\hline WT & Wild-type Synechocystis PCC 6803 & [14] \\
\hline pEEK2-ST & pEEK2-(PtrcBCD-kivd $\left.\boldsymbol{d}^{5286 T}-\mathrm{T}\right)-\mathrm{Km}^{\mathrm{R}}$ & [15] \\
\hline HXO & $\Delta d d h:: \mathrm{Cm}^{R}, \mathrm{pEEK} 2-\left(\mathrm{PtrcBCD}-\boldsymbol{k} \boldsymbol{i v} \boldsymbol{d}^{5286 T_{-T}}\right)-\mathrm{Km}^{\mathrm{R}}$ & This study \\
\hline $\mathrm{HX} 1$ & $\Delta d d h::\left(P p s b A 2-\boldsymbol{k i v}_{\boldsymbol{v}}{ }^{5 \mathbf{2 8 6 T}}-\mathrm{T}\right)-\mathrm{Cm}^{\mathrm{R}}, \mathrm{pEEK2}-\left(\mathrm{PtrcBCD}-\boldsymbol{k} \boldsymbol{i v} \boldsymbol{d}^{\mathbf{5 2 8 6 T}}-\mathrm{T}\right)-\mathrm{Km}^{\mathrm{R}}$ & This study \\
\hline $\mathrm{H} \times 2$ & 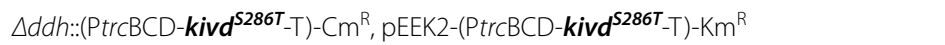 & This study \\
\hline $\mathrm{HX3}$ & 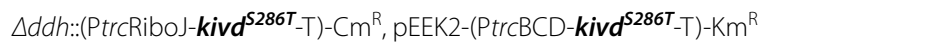 & This study \\
\hline $\mathrm{HX5}$ & $\Delta d d h::(P p s b A 2-a l s S-T)-C m^{R}$, pEEK2-(PtrcBCD-kivd $\left.\boldsymbol{d}^{5286 T}-\mathrm{T}\right)-\mathrm{Km}^{\mathrm{R}}$ & This study \\
\hline HX6 & 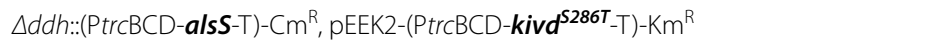 & This study \\
\hline HX7 & 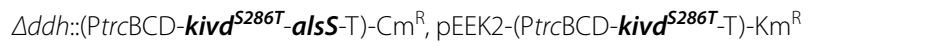 & This study \\
\hline HX8 & 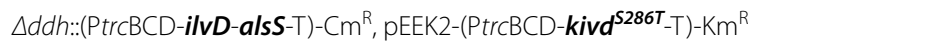 & This study \\
\hline HX9 & 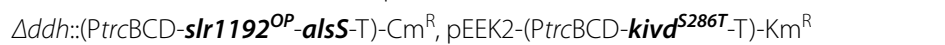 & This study \\
\hline HX15 & 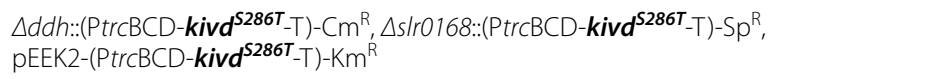 & This study \\
\hline HX16 & 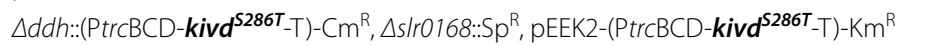 & This study \\
\hline $\mathrm{HX} 17$ & 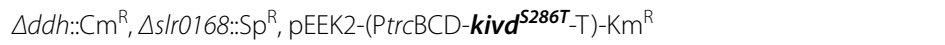 & This study \\
\hline $\mathrm{H} \times 28$ & 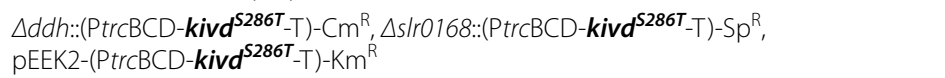 & This study \\
\hline HX29 & 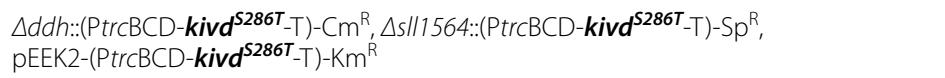 & This study \\
\hline HX39 & 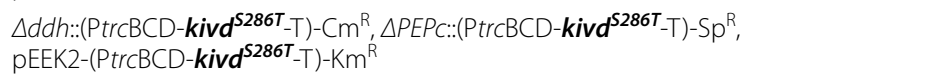 & This study \\
\hline $\mathrm{HX} 40$ & 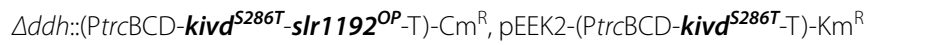 & This study \\
\hline $\mathrm{HX} 42$ & $\begin{array}{l}\Delta d d h::\left(P \operatorname{trcBCD}-s / r 1192^{O P}-a l s S-T\right)-C m^{R}, \Delta s / r 0168:(P \operatorname{trcBCD}-i l v C-i l v D-T)-S p^{R} \\
\text { pEEK2-(PtrcBCD-kivd }\end{array}$ & This study \\
\hline HX43 & 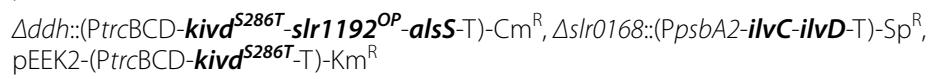 & This study \\
\hline $\mathrm{HX} 44$ & 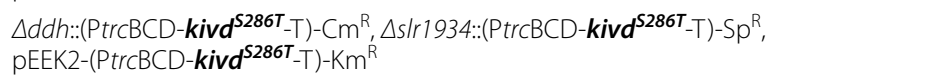 & This study \\
\hline HX45 & 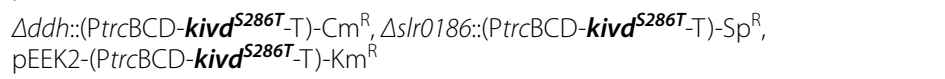 & This study \\
\hline HX46 & 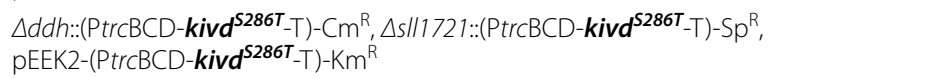 & This study \\
\hline $\mathrm{HX} 47$ & 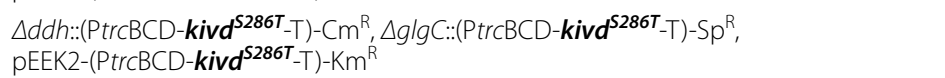 & This study \\
\hline
\end{tabular}

Expressed genes in bold

${ }^{a} \mathrm{Km}^{\mathrm{R}}$, Kanamycin resistance cassette; $\mathrm{Sp}^{\mathrm{R}}$, spectinomycin resistance cassette; $\mathrm{Cm}^{\mathrm{R}}$ : chloramphenicol resistance cassette; $\mathrm{T}$, Terminator BBa_B0015

$2 \mathrm{~mL}$ of PBS and centrifuged again at $5000 \mathrm{rpm} / 2400 \times g$ for $10 \mathrm{~min}$ at room temperature. The pellets were resuspended in $200 \mu \mathrm{L}$ of PBS and were frozen in $-80{ }^{\circ} \mathrm{C}$ for $10 \mathrm{~min}$ followed by heating at $37{ }^{\circ} \mathrm{C}$ for $10 \mathrm{~min}$. For the following steps, the samples were kept on ice. Four microliters of 50X Protease Arrest (GBioscience) and acid-washed glass beads (425-600 $\mu$ m diameter, SigmaAldrich) were mixed with cells, which were disrupted by using the Precellys-24 Beadbeater (Bertin Instruments) with $5000 \mathrm{rpm}$ program $4 \times 30 \mathrm{~s}$. After adding $100 \mu \mathrm{L}$ of PBS, the total lysates were centrifuged twice at $1976 \mathrm{rpm} / 1000 \times g, 4{ }^{\circ} \mathrm{C}, 30 \mathrm{~s}$, to obtain a transparent supernatant containing soluble proteins. The protein concentrations were determined by the DC protein assay (Bio-Rad).

Five micrograms (Strep-tagged proteins) and $20 \mu \mathrm{g}$ (His-tagged and Flag-tagged proteins) of soluble proteins were loaded and separated on sodium dodecyl sulfate polyacrylamide gel electrophoresis (SDS-PAGE), using Mini-PROTEAN TGX ${ }^{\mathrm{TM}}$ gels (Bio-Rad), and transferred to PVDF membranes (Bio-Rad). Proteins were detected using anti-Strep-tag (abcam, ab76949), anti-Flag-tag (Sigma-Aldrich, F3165) and anti-His-tag (GenScript, A00186) primary antibodies and HRPconjugated secondary antibodies: goat-anti-rabbit lgG (Bio-Rad, 972-4446) for Strep-tag; rabbit-antimouse lgG (Agrisera, AS101114) for both Flag-tag and 
His-tag. Bands were detected using the Clarity ECL substrate (Bio-Rad) and quantified using Quantity One Software (Bio-Rad).

\section{Optical density measurement and isobutanol extraction} Cell growth was monitored by measuring optical density at $750 \mathrm{~nm}\left(\mathrm{OD}_{750}\right)$ of each cell culture. The absorbance at $750 \mathrm{~nm}$ was measured every day for $200 \mu \mathrm{L}$ cell culture in 96-well plate (SARSTEDT) using a microplate reader (HIDEX, Plate Chameleon).

Every second day, $2 \mathrm{~mL}$ of cell culture were sampled from each flask and centrifuged at $5000 \mathrm{rpm} / 2400 \times g$ for $10 \mathrm{~min}$. Then, $1305 \mu \mathrm{L}$ of supernatant were transferred into a $15 \mathrm{~mL}$ screw cap tube and mixed with $45 \mu \mathrm{L}$ of $3000 \mathrm{mg} \mathrm{L}^{-1}$ internal standard 1-pentanol (Sigma-Aldrich) and $450 \mu \mathrm{L}$ of extraction solvent dichloromethane (DCM, Sigma-Aldrich). The mixture was shaken on Multi-tube Vortexer VX-2500 (VWR) at maximal speed for $5 \mathrm{~min}$, followed by centrifugation at $5000 \mathrm{rpm} / 4500 \times \mathrm{g}, 4{ }^{\circ} \mathrm{C}$, for $10 \mathrm{~min}$. DCM phase was then transferred into $1.5 \mathrm{~mL}$ clear glass gas chromatography vials (VWR) for gas chromatography analysis.

\section{Isobutanol quantification by gas chromatography}

A detailed protocol for isobutanol quantification has been described [14]. In short, the extracted samples were analyzed on a PerkinElmer GC 580 system equipped with a flame ionization detector (FID) and an EliteWAX Polyethylene Glycol Series Capillary column, $30 \mathrm{~m} \times 0.25 \mathrm{~mm} \times 0.25 \mu \mathrm{m}$ (PerkinElmer). Nitrogen was the carrier gas with a rate of $10 \mathrm{~mL} \mathrm{~min}{ }^{-1}$. The temperatures of injector and detector were $220^{\circ} \mathrm{C}$ and $240{ }^{\circ} \mathrm{C}$, respectively. Obtained GC results were analyzed using TotalChrom Navigator version 6.3.2.

\section{Results and discussion}

Expressing AlsS has an effect on isobutanol biosynthesis Acetolactate synthase (AlsS), catalyzing condensation of pyruvate into 2-acetolactate, is the first enzyme of the 2-keto acid pathway, which plays a vital role in converting more carbon from the central metabolite pyruvate towards isobutanol synthesis. It has been shown that overexpression of als $S$ contributed to a 1.8-fold improvement of isobutanol production in B. subtilis [10]. On the other hand, AlsS functions not only as acetolactate synthase to condense two pyruvate molecules, but also it was experimentally verified that it functions as $\alpha$-ketoisovalerate decarboxylase [20]. Previously, $\alpha$-ketoisovalerate decarboxylase was identified as the bottleneck in the isobutanol synthesis pathway in Synechocystis cells [12]. Here, the effects of expressing AlsS on isobutanol production was explored in isobutanolproducing Synechocystis strains.
It has been reported to be challenging to get successful transformants containing AlsS, not only in Synechocystis [12], but also in C. cellulolyticum [9], possibly due to the high activity of AlsS [9]. In the present study, instead of being placed on self-replicating plasmid, alsS was integrated in the chromosome of Synechocystis for expression. To increase the probability of successfully generating Synechocystis transformants containing AlsS, AlsS expression was controlled by a selection of promoters with varied strength and organization of gene orders within the operon. HX0 was generated as a control strain, with chloramphenicol resistance $\left(\mathrm{Cm}^{\mathrm{R}}\right)$ cassette integrated into the $d d h$ site and Strep-kivd ${ }^{S 286 T}$ placed on a self-replicating plasmid (Fig. 2A). In HX5, PpsbA2 was used to drive alsS expression (Fig. 2A). As expected, when changing from PpsbA2 to PtrcBCD, AlsS could not be detected by Western-immunoblot (Additional file 1: Fig. S1). Spontaneous mutation(s) of AlsS may occur due to its high activity [9]. In addition, alsS was expressed with either Flag-kivd ${ }^{S 286 T}$, ilvD or $\operatorname{slr} 1192^{O P}$ as an operon, to generate strains HX7, HX8, and HX9 (Fig. 2A). In all strains, apart from integrating selected genes into the $d d h$ site of chromosome, Strep-kivd ${ }^{S 286 T}$ was placed on self-replicating plasmid for expression (Fig. 2A). AlsS was successfully detected in all four engineered strains by Western-immunoblot (Fig. 2D). This is the first report demonstrating that alsS was integrated into Synechocystis chromosome, and its expression was successfully confirmed by Western-immunoblot (Fig. 2D). Unexpectedly, none of the four engineered strains produced significantly higher isobutanol than the control strain HX0 (Fig. 2C).

With almost the same isobutanol production per cell achieved by strains HXO, 7, 8 and 9 (Fig. 2C), it is interesting to observe that the Strep_Kivd ${ }^{\mathrm{S} 286 \mathrm{~T}}$ expression level was not consistent in the four strains, and a significant decrease of Strep-Kivd ${ }^{\mathrm{S} 286 \mathrm{~T}}$ protein level was detected in strains HX7, 8 and 9 (Fig. 2D; Additional file 1: Table S4).

The broad host range RSF1010 replicon-based selfreplicating plasmid is widely used when demonstrating biofuel production in cyanobacteria [18, 21, 22]. However, this is the first study expressing genes with both a self-replicating plasmid-based system and a genomebased system in Synechocystis cells. Interestingly, selfreplicating plasmid-based system may not be as stable as expected. When a heterologous gene is integrated in the chromosome, the expression level of a heterologous gene on a self-replicating plasmid is affected dramatically (Fig. 2D; Additional file 1: Table S4). In our previous study, it was observed that isobutanol production was positively correlated with Kivd $^{\mathrm{S} 286 \mathrm{~T}}$ expression level [12]. However, in the present study, similar isobutanol production was observed (Fig. 2C), though less 


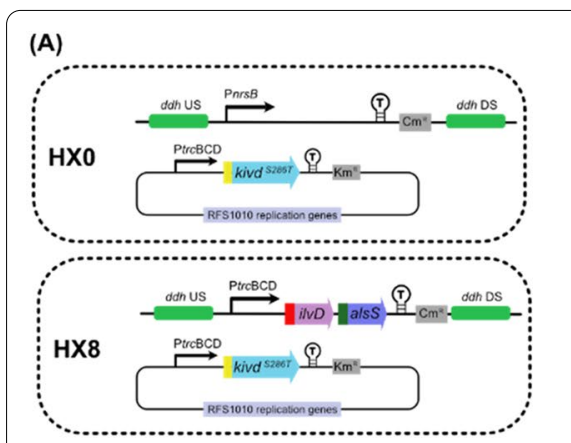

(B)

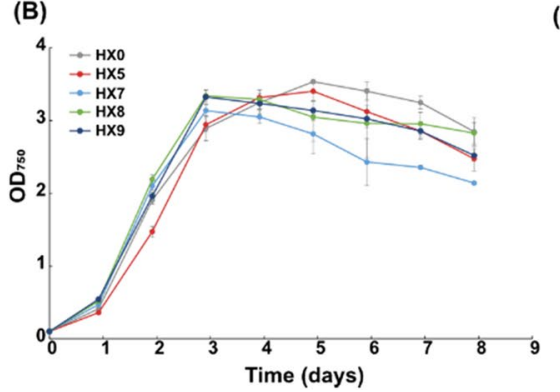

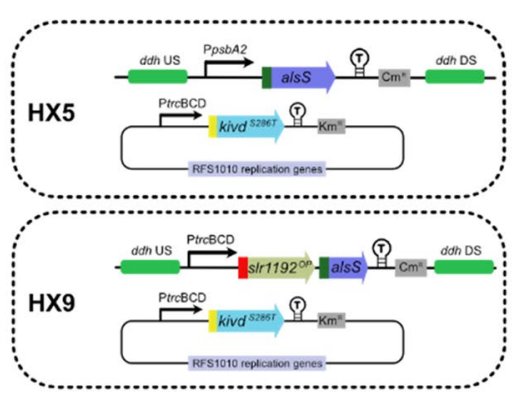

(C)

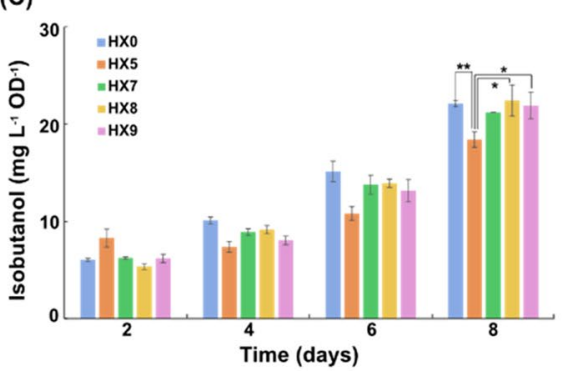

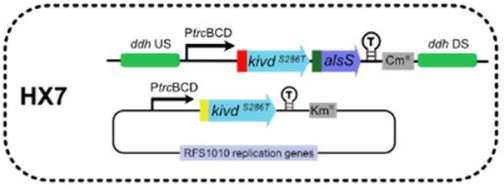

(D)

SDS-PAGE L $\quad$ HXO:HX5:HX7:HX8:HX9

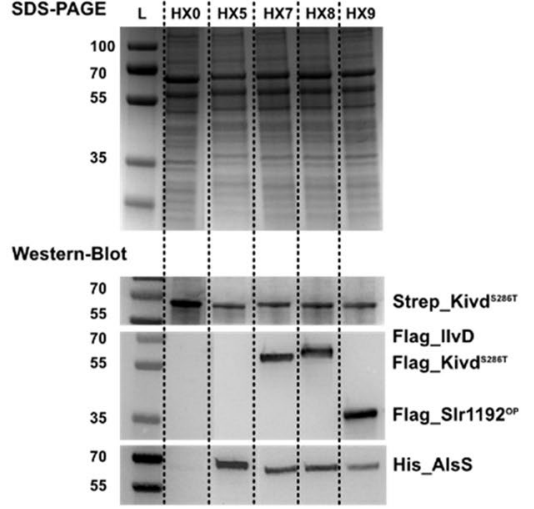

Fig. 2 Schematic overview of genetic constructs used and comparison of growth, protein levels (SDS-PAGE/Western-immunoblot) and isobutanol production per cell in engineered Synechocystis PCC 6803 strains HX0, HX5, HX7, HX8, and HX9. A Schematic presentation of the genetic constructs in the engineered strains. kivd ${ }^{5286 T}$ : encodes a-ketoisovalerate decarboxylase (Lactococcus lactis); alsS: encodes acetolactate synthase (Bacillus subtilis); ilvD: encodes dihydroxy-acid dehydratase (Escherichia coli); s/r1192 OP: encodes codon optimized alcohol dehydrogenase (Synechocystis). Kivd $^{\text {S286T }}$ expressed on self-replicating plasmid was Strep-tagged at the N-terminal; Kivd ${ }^{\text {S286T }}$, IlvD, and SIr1192 ${ }^{\mathrm{OP}}$ expressed in the ddh (s/r1556) site of chromosome was Flag-tagged at the N-terminal; AlsS expressed in the $d d h$ (s/r1556) site of chromosome was His-tagged at the N-terminal. B Growth curves of the engineered strains during 8-day cultivation. C Isobutanol production per cell at day 2, 4, 6, and 8 of indicated strains. D SDS-PAGE (top) and Western-immunoblot (bottom). Each lane represents result from respective strain. L: ladder (in kDa). For SDS-PAGE, 20 $\mu$ g of total soluble protein were loaded for each strain. For Western-immunoblot, $5 \mu \mathrm{g}, 20 \mu \mathrm{g}$, and $20 \mu \mathrm{g}$ of total soluble protein were loaded for each strain to detect Strep-tagged, Flag-tagged, and His-tagged protein, respectively. Results are the mean of three biological replicates, each with three technical replicates. Error bars represent standard deviation. Asterisk represents significant difference between different strains (one-way ANOVA, $\left.{ }^{*} \mathrm{p}<0.05,{ }^{* *} \mathrm{p}<0.005\right)$

Strep-Kivd ${ }^{\text {S286T }}$ is expressed in the engineered strains HX7-9 compared to the control strain HXO (Fig. 2D; Additional file 1: Table S4). Therefore, additional expression of AlsS may make up for the decreased isobutanol production as a result of the decreased expression level of Strep-Kivd ${ }^{\mathrm{S} 286 \mathrm{~T}}$, enabling the engineered strains to reach similar isobutanol production per cell as the control strain. On the other hand, different from other engineered strains, HX5 grew slower between days 0 and 3 (Fig. 2B; Additional file 1: Fig. S2) and produced less isobutanol per cell than control strain HX0 (Fig. 2C), which may be explained by the significantly decreased expression of Kivd ${ }^{\text {S286T }}$ (Fig. 2D; Additional file 1: Table S4). When compared with strains HX8-9, HX5 produced less isobutanol (Fig. 2C), although a higher expression level of AlsS was observed in HX5 (Fig. 2D; Additional file 1: Table S4), indicating that IlvD and Slr1192 ${ }^{\mathrm{OP}}$ are important enzymes for further enhancement of isobutanol production in Synechocystis.

\section{Complete 2-keto acid pathway integration enhances isobutanol production}

As discussed above, similar isobutanol production per cell was observed in strains with $\mathrm{Kivd}^{\mathrm{S} 286 \mathrm{~T}}$ solely expressed or Kivd ${ }^{\mathrm{S} 286 \mathrm{~T}}$ expressed together with one or two selected enzymes from 2-keto acid pathway (Fig. 2C). Integrating kivd from L. lactis and $y q h D$ from E. coli into the neutral site I (NSI) of the Synechococcus chromosome resulted in a production of $18 \mathrm{mg}$ isobutanol $\mathrm{L}^{-1}$ [11]. After further integrating alsS from B. subtilis and $i l v C$ and $i l v D$ from E. coli, the final Synechococcus strain produced $450 \mathrm{mg}$ isobutanol $\mathrm{L}^{-1}$, a 25 -fold improvement [11]. Therefore, in order to further increase isobutanol production, integrating a complete 2-keto acid pathway into Synechocystis cell may be a promising strategy. Our further engineering of Synechocystis use AlsS from B. subtilis, IlvC and IlvD from E. coli, the engineered version of Kivd (Kivd ${ }^{\mathrm{S} 286 \mathrm{~T}}$ ) [15], and the codon optimized version of Slr1192 (Slr1192 ${ }^{\mathrm{OP}}$ ) [23]. With five selected enzymes, two engineered strains with a complete 2-keto acid pathway integrated were generated, HX42 and HX43. In 
strain HX42, slr1192 ${ }^{O P}$ and alsS were integrated into the $d d h$ site and expression was driven by strong promoter Ptrc, ilvC and $i l v D$ were integrated into the slr0168 site and expression was driven by strong promoter Ptrc, and Strep-kivd ${ }^{S 286 T}$ was placed on self-replicating plasmid and expression was driven by strong promoter Ptrc (Fig. 3A). In strain HX43, Flag-kivd ${ }^{S 286 T}$, alsS and slr1192 ${ }^{O P}$ were integrated into the $d d h$ site and expression was driven by strong promoter Ptrc, ilvC and $i l v D$ were integrated into the slr0168 site and expression was driven by native promoter PpsbA2, and Strep-kivd ${ }^{S 286 T}$ was placed on selfreplicating plasmid and expression was driven by strong promoter Ptrc (Fig. 3A). Meanwhile, strains HX17 and HX16 were generated as control strains for HX42 and HX43, respectively (Fig. 3A). For HX17, chloramphenicol resistance $\left(\mathrm{Cm}^{\mathrm{R}}\right)$ cassette was integrated into the $d d h$ site, spectinomycin resistance $\left(\mathrm{Sp}^{\mathrm{R}}\right)$ cassette was integrated into the slr0168 site, and Strep-kivd ${ }^{S 286 T}$ placed on self-replicating plasmid. For HX16, Flag-kivd ${ }^{\text {S286T }}$ was integrated into the $d d h$ site, spectinomycin resistance

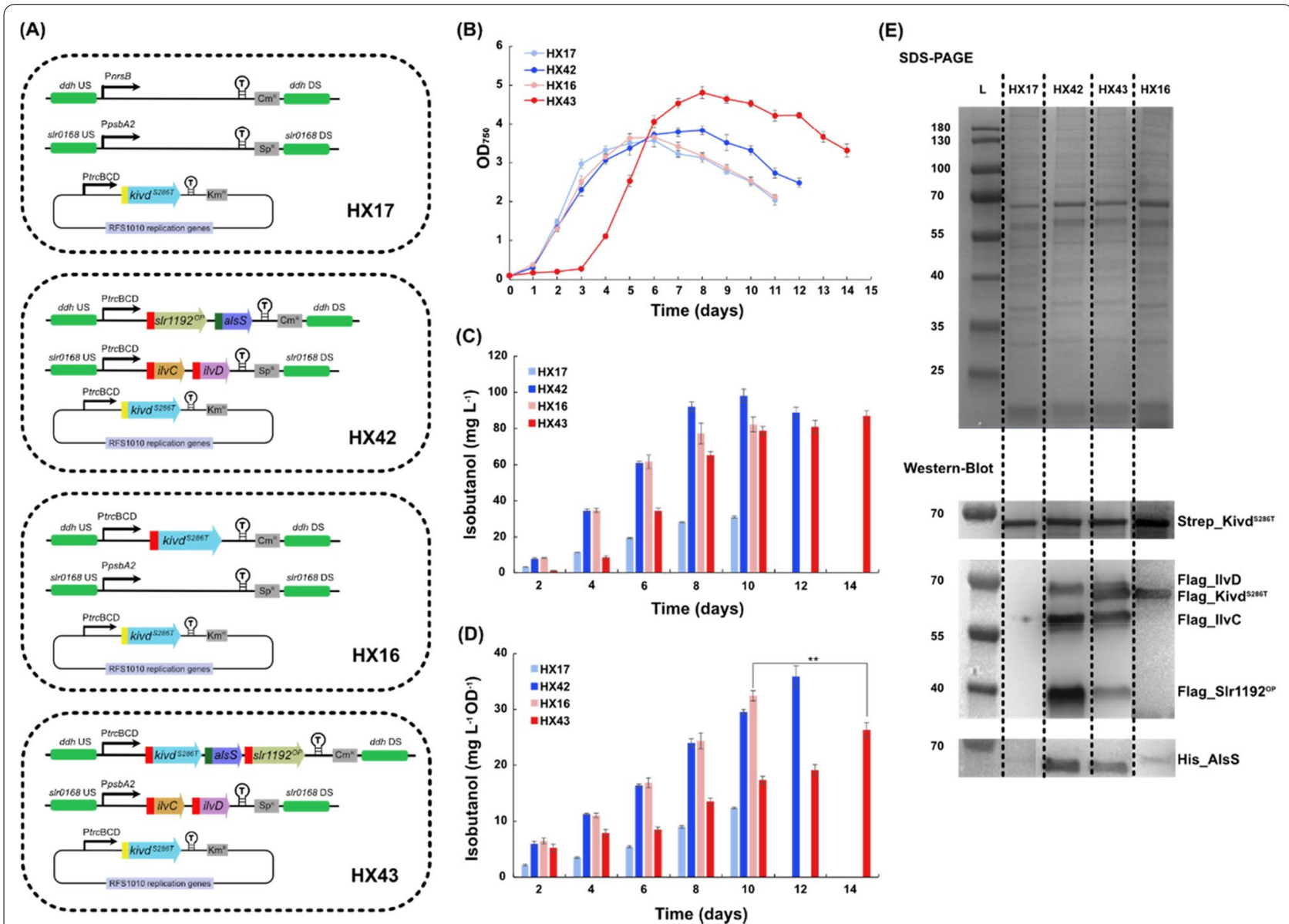

Fig. 3 Schematic overview of genetic constructs used and comparison of growth, protein levels (SDS-PAGE/Western-immunoblot) and isobutanol production/isobutanol production per cell in engineered Synechocystis PCC 6803 strains HX17, HX42, HX16 and HX43. A Schematic presentation of the genetic constructs in the engineered strains. kivd ${ }^{5286 T}$ : encodes a-ketoisovalerate decarboxylase (Lactococcus lactis); alsS: encodes acetolactate synthase (Bacillus subtilis); ilvC: encodes acetohydroxy-acid isomeroreductase (Escherichia coli); ilvD: encodes dihydroxy-acid dehydratase (E. coli); s/r1192 ${ }^{\mathrm{OP}}$ : encodes codon optimized alcohol dehydrogenase (Synechocystis). HX16 and HX17 were generated as control strains. Kivd ${ }^{5286 \mathrm{~T}}$ expressed on self-replicating plasmid was Strep-tagged at the N-terminal; IIvC, IIvD, SIr1192 ${ }^{\mathrm{OP}}$, and Kivd ${ }^{\mathrm{S} 286 \mathrm{~T}}$ expressed in either the $d d h$ ( $\operatorname{sir} 1556$ ) site or the s/r0168 site of chromosome was Flag-tagged at the N-terminal. AlsS expressed in the $d d h$ (s/r1556) site of chromosome was His-tagged at the N-terminal. B Growth curves of the engineered strains during 14-day cultivation. C Isobutanol production of indicated strains. D Isobutanol production per cell of indicated strains. E SDS-PAGE (top) and Western-immunoblot (down). Each lane represents result from respective strain. L: ladder (in kDa). For SDS-PAGE, $5 \mu \mathrm{g}$ of total soluble protein were loaded for each strain. For Western-immunoblot, $5 \mu \mathrm{g}, 20 \mu \mathrm{g}$, and $20 \mu \mathrm{g}$ of total soluble protein were loaded for each strain to detect Strep-tagged, Flag-tagged, and His-tagged protein, respectively. Results are the mean of three biological replicates, each with three technical replicates. Error bars represent standard deviation. Asterisk represents significant difference between different strains (one-way ANOVA, ${ }^{* *} p<0.005$ ) 
$\left(\mathrm{Sp}^{\mathrm{R}}\right)$ cassette was integrated into the slr0168 site, and Strep-kivd ${ }^{S 286 T}$ placed on self-replicating plasmid.

Strain HX42 grew slightly slower than HX17 between days 2 and 5 (Fig. 3B). However, after day 6, the $\mathrm{OD}_{750}$ of HX17 started to decline while the $\mathrm{OD}_{750}$ of $\mathrm{HX} 42$ continued increasing until day 8 before declining from day 9 (Fig. 3B). Unexpectedly, HX43 had a much longer growth lag, with practically no cell growth observed during the first three days (Fig. 3B). However, thereafter HX43 started to grow and reached a highest density $\left(\mathrm{OD}_{750}=4.8\right)$ on day 8 (Fig. 3B). Both strains HX42 and HX43 contain a complete 2-keto acid pathway, thus the distinct growth pattern of HX43 may due to the different expression units, e.g. promoters, and gene order within the operons (Fig. 3A).

The first engineered Synechocystis strain with a complete 2-keto acid pathway integrated, HX42, produced $98 \mathrm{mg}$ isobutanol $\mathrm{L}^{-1}$ at day 10 , while the control strain HX17 produced $31 \mathrm{mg} \mathrm{L}^{-1}$ (Fig. 3C). The expression of all five enzymes were confirmed by Western-immunoblot
(Fig. 3E) with a 190\% increase of isobutanol production per cell compared to the control strain HX17 (Fig. 3D). Interestingly, even though strains HX17 and HX0 contain the same Strep-kivd ${ }^{S 286 T}$ placed on self-replicating plasmid (Figs. 3A, 4A), the resulting isobutanol production of HX17 was only half of that of HX0 (Figs. 3C, 4C). A most likely reason is the different expression levels of Strep$\mathrm{Kivd}^{\mathrm{S} 286 \mathrm{~T}}$ (Fig. 2D, Fig. 3E; Additional file 1: Table S4). When compared to HXO, the difference of HX17 is that slr0168 gene was replaced with an additional spectinomycin resistance $\left(\mathrm{Sp}^{\mathrm{R}}\right)$ cassette.

As $\mathrm{Kivd}^{\mathrm{S} 286 \mathrm{~T}}$ has been identified as a bottleneck for isobutanol synthesis [12, 14], an additional copy, Flag $k_{i v d} d^{S 286 T}$, was integrated into the $d d h$ site in an operon together with alsS and $s l r 1192^{O P}$, in order to increase the total expression of $\mathrm{Kivd}^{\mathrm{S} 286 \mathrm{~T}}$ (strain HX43). HX43 produced $87 \mathrm{mg}$ isobutanol $\mathrm{L}^{-1}$, which is similar to control strain HX16 (Fig. 3C). However, the isobutanol production per cell in HX43 was lower than in control strain HX16 (Fig. 3D), due to the higher optical density $\left(\mathrm{OD}_{750}\right)$
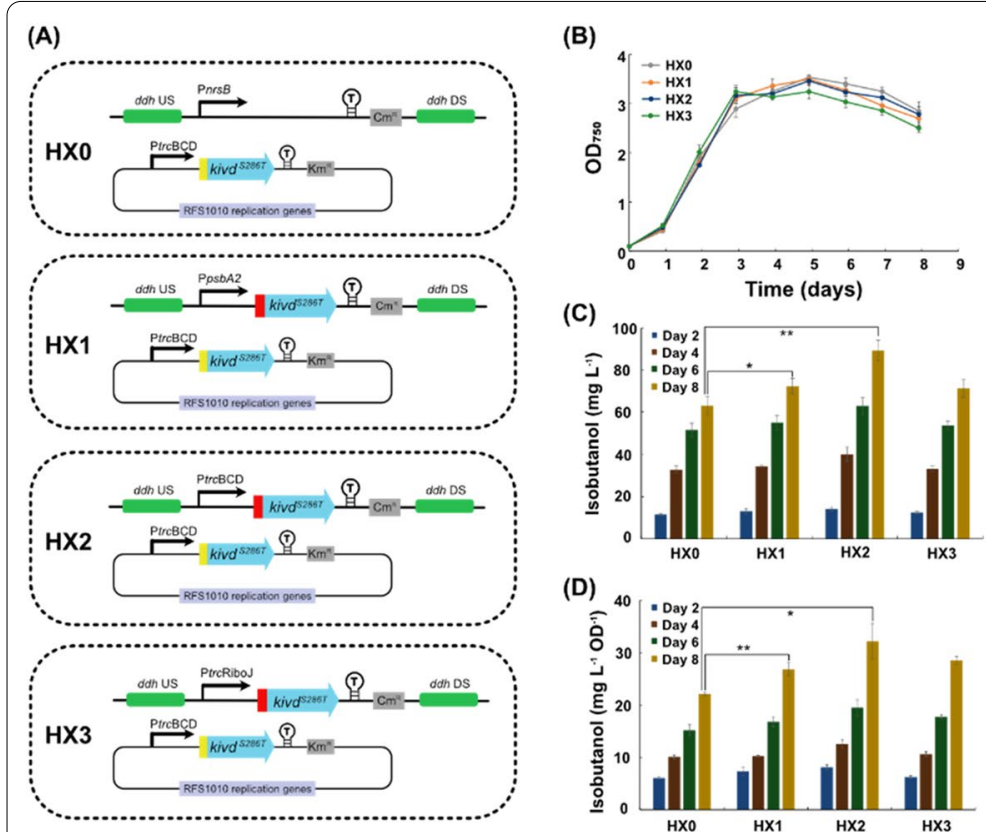

(E)
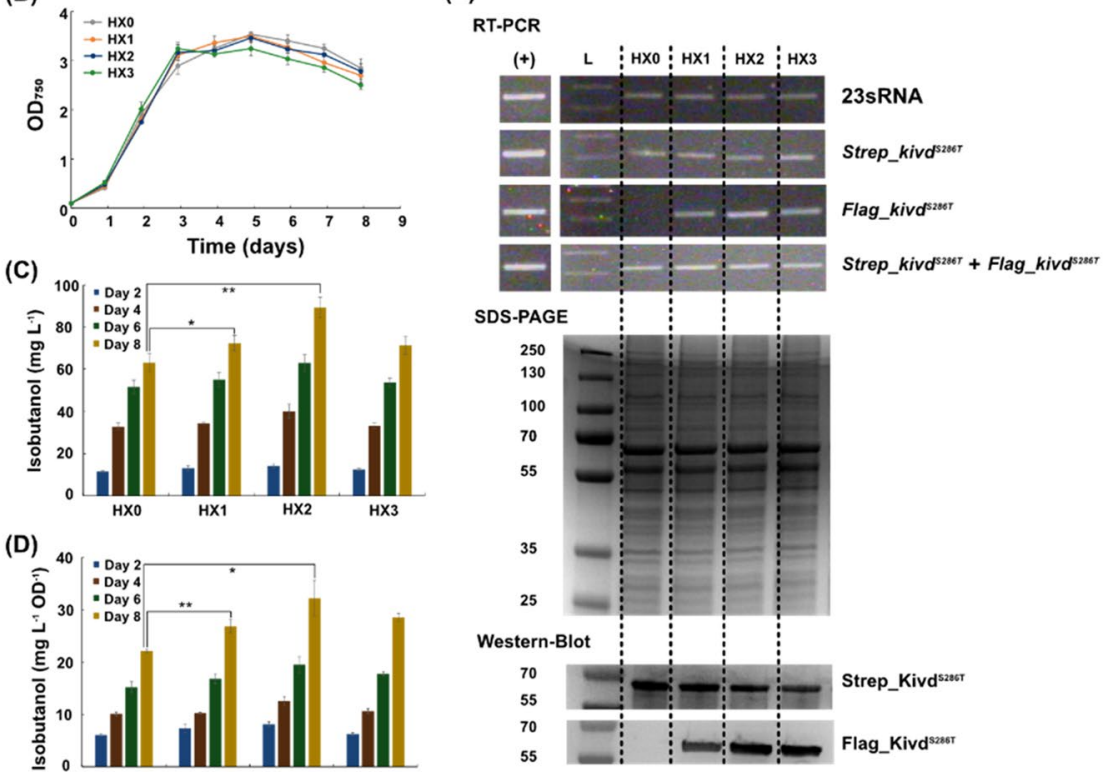

Fig. 4 Schematic overview of genetic constructs used and comparison of growth, relative gene transcript (RT-PCR), protein levels (SDS-PAGE/ Western-immunoblot) and isobutanol production/isobutanol production per cell in engineered Synechocystis PCC 6803 strains HX0, HX1, HX2, and HX3. A Schematic presentation of the genetic constructs in the engineered strains. kivd ${ }^{5286 T}$ : encodes a-ketoisovalerate decarboxylase (Lactococcus lactis). $\mathrm{HX1}, 2,3$ contain two copies of kivd ${ }^{5286 T}$ : first copy was put on a self-replicating plasmid and its expression was driven by PtrcBCD; second copy was integrated in the $d d h$ (s/r1556) site of chromosome and its expression was driven by PpsbA2, PtrcBCD and PtrcRiboJ, respectively. HXO was generated as a control strain. Kivd ${ }^{5286 T}$ expressed on self-replicating plasmids was Strep-tagged at the N-terminal; Kivd ${ }^{5286 T}$ expressed in the ddh (s/r1556) site of chromosome was Flag-tagged at the N-terminal. B Growth curves of the engineered strains during 8-day cultivation. C Isobutanol production at day 2, 4, 6, and 8 of indicated strains. D Isobutanol production per cell at day 2, 4, 6, and 8 of the indicated strains. E RT-PCR (top), SDS-PAGE (middle) and Western-immunoblot (bottom). Each lane represents result from respective strain. L: ladder (in kDa); (+): positive control, RT-PCR performed using Synechocystis genomic DNA or corresponding plasmid as template. For SDS-PAGE, $20 \mu \mathrm{g}$ of total soluble protein were loaded for each strain. For Western-immunoblot, $5 \mu \mathrm{g}$ and $20 \mu \mathrm{g}$ of total soluble protein were loaded for each strain to detect Strep-tagged Kivd ${ }^{5286}$ and Flag-tagged Kivd ${ }^{5286 T}$, respectively. Results are the mean of three biological replicates, each with three technical replicates. Error bars represent standard deviation. Asterisk represents significant difference between different strains (one-way ANOVA, ${ }^{*} p<0.05,{ }^{* *} p<0.005$ ) 
of HX43 from day 6 until the end of cultivation (Fig. 3B). As shown in Western-immunoblot (Fig. 3E), the higher isobutanol production per cell of HX16 may be explained by the higher total expression level of $\mathrm{Kivd}^{\mathrm{S} 286 \mathrm{~T}}$ (combined levels of Strep_Kivd ${ }^{\text {S286T }}$ and Flag_Kivd ${ }^{\text {S286T }}$ ) in HX16 compared to in HX43. Moreover, after integration of the Flag kivd $d^{S 286 T}$ into the $d d h$ site, the expression level of the other introduced genes in HX43 decreased significantly compared to in strain HX42 (Fig. 3E; Additional file 1: Table S4). As a consequence, the expression of the other enzymes of the 2-keto acid pathway may not have been enough to compensate for the decreased isobutanol production as a result from the decreased total Kivd $^{\text {S286T }}$ expression level.

\section{Kivd $^{\text {S286T }}$, an identified rate-limiting enzyme in 2-keto acid pathway for isobutanol synthesis}

Our earlier study showed that engineered Synechocystis strain with $k i v d^{S 286 T}$ expressed on pEEK2, a self-replicating plasmid carrying the RSF1010 replicon, reached the highest isobutanol production [12]. As a key enzyme for isobutanol biosynthesis in Synechocystis, protein expression level of Kivd ${ }^{\mathrm{S} 286 \mathrm{~T}}$ was demonstrated to be positively correlated with isobutanol production, and was regarded as the main bottleneck for further improvement of isobutanol production. Thus, in parallel with integration of a complete 2-keto acid pathway into Synechocystis cells, some efforts were invested to increase the expression level of $\mathrm{Kivd}^{\mathrm{S} 286 \mathrm{~T}}$ in the cells with the aim of potentially enhancing isobutanol production.

Various approaches have been developed and applied to increase protein expression levels in Synechocystis cells [23-26]. Increasing gene dosage is an efficient approach to increase both gene transcription and translation, which has been successfully applied in Synechocystis for ethanol production [24], and in Synechococcus for squalene production [27]. 5'-region optimizations, including a selection of both promoters and genetic insulators, was systematically applied in Synechocystis resulting in significantly improved 1-butanol production [23].

Apart from placing one copy of kivd ${ }^{S 286 T}$ (Strep_ $k_{i v d^{S 286 T}}$ ) on pEEK2, a second copy (Flag_kivd ${ }^{S 286 T}$ ) was integrated into the $d d h$ site of chromosome, to generate strain HX1 harboring two gene copies (Fig. 4A). The expression of Flag_kivd ${ }^{S 286 T}$ was driven by PpsbA2, a native light intensity-dependent promoter [28]. HX0 was constructed as a control strain, with a single copy of Strep_kivd $d^{S 286 T}$ placed on pEEK2 and $\mathrm{Cm}^{\mathrm{R}}$ cassette integrated into the $d d h$ site (Fig. 4A). Similar growth patterns were observed for HX0 and HX1 (Fig. 4B), indicating gene dosage of kivd $d^{S 286 T}$ did not compromise cell growth. At day 8, isobutanol production of $\mathrm{HX} 0$ reached $63 \mathrm{mg} \mathrm{L}^{-1}$ (Fig. 4C) and strain HX1 was able to produce
$72 \mathrm{mg}$ isobutanol $\mathrm{L}^{-1}$ (Fig. 4C), which was $14 \%$ improvement compared to HX0. Similarly, a moderate increase of isobutanol production per cell was observed for HX1, up to $23 \%$ (Fig. 4D). This significant but still not dramatic increase may be explained by two factors: first, pEEK2 contains RSF1010 replicon which has a higher copy number (between 10 and 30) [29] than the Synechocystis chromosome (around 12) [30]; second, the promoter PpsbA2 is weaker in driving Kivd $^{\mathrm{S} 286 \mathrm{~T}}$ expression compared to Ptrc [14].

In strain HX1, PpsbA2 was used to drive Flag$k i v d^{S 286 T}$ expression in the $d d h$ site of chromosome. To attain higher Flag_Kivd ${ }^{\text {S286T }}$ protein expression for further isobutanol production improvement, PpsbA2 was replaced with the stronger promoter Ptrc, coupled with a BCD (bicistronic design) [31] or a RiboJ (self-cleaving ribozyme) [32], generating strains $\mathrm{HX} 2$ and $\mathrm{HX} 3$ (Fig. 4A). As expected, the translation level of Flagkivd $^{S 286 T}$ increased in HX2 and HX3, compared to HX1 (Fig. 4E; Additional file 1; Table S4). Both transcription and translation levels of Flag_kivd ${ }^{S 286 T}$ in HX2 was higher than in HX3 (Fig. 4E; Additional file 1: Table S4), indicating that the BCD element is more suitable to improve Flag-Kivd ${ }^{\text {S286T }}$ expression level. As the best performing strain, after 8-day cultivation, the isobutanol production and isobutanol production per cell of strain $\mathrm{HX} 2$ reached $89 \mathrm{mg} \mathrm{L}^{-1}$ and $32 \mathrm{mg} \mathrm{L}^{-1} \mathrm{OD}^{-1}, 42 \%$ and $46 \%$ increase respectively, compared to those achieved by $\mathrm{HX0}$ (Fig. 4C, D). As for strain $\mathrm{HX} 3$, its isobutanol production and isobutanol production per cell reached similar level as HX1 (Fig. 4C, D), which may be explained by the similar total $\mathrm{Kivd}^{\mathrm{S} 286 \mathrm{~T}}$ protein levels in both strains. Even though more Flag_Kivd ${ }^{\mathrm{S} 286 \mathrm{~T}}$ expressed in HX3 than that expressed in HX1, less Strep_Kivd ${ }^{\text {S286T }}$ was expressed in HX3 than expressed in HX1, resulting in similar total Kivd $^{\mathrm{S} 286 \mathrm{~T}}$ protein expression level. The transcriptions of all kivd ${ }^{S 286 T}$ genes were confirmed using RT-PCR (Fig. 4E). The transcription level of Strep_kivd ${ }^{S 286 T}$ was similar in all four engineered strains, while strain HX2 showed the highest transcription level of Flag_kivd ${ }^{\text {S286T }}$ (Fig. 4E).

Both strategies of increasing gene dosage and replacement of a strong promoter resulted in improved isobutanol production in Synechocystis, which is consistent to what was observed for improved squalene production with the same strategies performed in Synechococcus [32]. After integrating a second copy of Flag-kivd ${ }^{S 286 T}$ in the $d d h$ site, strains HX1, 2 and 3 showed higher isobutanol production than the control strain, which was positively related to total $\mathrm{Kivd}^{\mathrm{S} 286 \mathrm{~T}}$ protein levels, as observed previously [12]. A decrease of Strep-Kivd ${ }^{\text {S286T }}$ protein expression was detected by Western-immunoblot in strains HX1, 2 and 3 (Fig. 4E; Additional file 1: Table S4). 
Two hypotheses could be made. First, when introducing a second copy of Flag-kivd ${ }^{S 286 T}$ in the chromosome, the copy numbers of the RSF1010 replicon-based plasmid declined, leading to less Strep-kivd ${ }^{S 286 T}$ gene dosage contributed from the self-replicating plasmid and resulting decreased Strep-Kivd ${ }^{\text {S286 }}$ expression. Second, Flag-kivd $^{S 286 T}$ in the chromosome and Strep-kivd ${ }^{S 286 T}$ on self-replicating plasmid having identical gene sequences, encoding the same heterologous protein, may interfere with each other at transcription and translation level. This may lead to decreased protein expression level [33]. However, there was no significant difference of mRNA relative levels of Strep-kivd ${ }^{\text {S286T }}$ being observed between the four strains (Fig. 4E). This indicates similar mRNA levels of Strep-kivd ${ }^{S 286 T}$ on the self-replicating plasmid, supporting an interference on the translation level, but not on transcription, resulting in different protein expression levels.

Additional attempts were explored to improve $\mathrm{Kivd}^{\text {S286T }}$ expression levels. An effective approach improving $\beta$-phellandrene synthase protein expression has been demonstrated by fusing $\beta$-phellandrene synthase with phycocyanin $\beta$-subunit (encoded by $c p c B$ gene), resulting in a 100-fold improvement of $\beta$-phellandrene hydrocarbons production in Synechocystis [25]. Therefore, to potentially improve Kivd ${ }^{\text {S286 }}$ expression level, different $k i v d^{S 286 T}$ fusion constructs were generated with the Synechocystis endogenous $c p c B$ gene. However, no isobutanol production was detected (data not shown). Moreover, the small endogenous plasmid of Synechocystis, pCA2.4 is stable and has been shown as to have the potential to increase gene expression level [26]. However, only trace amount of isobutanol was detected after integrating $k i v d^{S 286 T}$ into a predicted neutral site of pCA2.4 (data not shown).

\section{Rewiring carbon flux by integrating third copy of kivd ${ }^{5286 T}$ in various sites of the chromosome}

After introducing a second copy of Flag-kivd $d^{5286 T}$, the resulting strain HX2 was able to achieve higher isobutanol production, confirming $\mathrm{Kivd}^{\mathrm{S} 286 \mathrm{~T}}$ as a key and rate-limiting enzyme in the 2-keto acid pathway for isobutanol synthesis. In strain HX2, it is unclear if Kivd ${ }^{\text {S286T }}$ is still the bottleneck, or the bottleneck is shifted to other enzymes in the isobutanol synthesis pathway (Fig. 1). Initially, in order to explore if Adh is the new bottleneck for isobutanol synthesis or not, strain HX40 was generated, with Flag-kivd ${ }^{S 286 T}$ and $\operatorname{slr} 1192^{O P}$ co-expressed as an operon in the $d d h$ site of chromosome (Fig. 5A). Similar growth patterns were observed for strains HX2 and HX40 (Fig. 5C), and protein expression was confirmed by Western-immunoblot (Fig. 5B). However, HX40 did not produced more isobutanol than strain HX2 (Fig. 5D), which with sole Flag-kivd ${ }^{S 286}$ integrated in the $d d h$ site of chromosome (Fig. 5A). Thus, the native alcohol dehydrogenases (encoded by slr1192 and slr0942) are not bottlenecks for isobutanol production in strain HX2. As expected, when co-expressed with $\operatorname{slr} 1192^{O P}$ in an operon in the chromosome, there was less Flag-Kivd ${ }^{\mathrm{S} 286 \mathrm{~T}}$ protein expressed (strain HX40) when compared with expressed solely in the chromosome (strain HX2), which is consistent with what was observed earlier [14]. Therefore, at present stage, isobutanol production is still positively correlated with the total $\mathrm{Kivd}^{\mathrm{S} 286 \mathrm{~T}}$ expression level, making Kivd $^{\mathrm{S} 286 \mathrm{~T}}$ a target for further investigations.

Therefore, more efforts were made to further increase the total Kivd ${ }^{\text {S286T }}$ expression level in Synechocystis cells. In strain HX2, a strong Ptrc, coupled with BCD was used to drive the expression of kivd ${ }^{S 286 T}$ on self-replicating plasmid as well as in the $d d h$ site of chromosome. It is challenging to further improve Kivd ${ }^{\mathrm{S} 286 \mathrm{~T}}$ expression by tuning transcription and translation through systematically screening different promoters as well as ribosome binding sites (RBSs). Instead, further increasing gene dosage of $k i v d^{S 286 T}$ may be an efficient and straightforward approach to achieve a higher total Kivd ${ }^{\mathrm{S} 286 \mathrm{~T}}$ expression level. The $d d h$ site was used for the introduction of the second copy of Flag-kivd ${ }^{S 286 T}$ in the previous step, which may be a competing pathway for isobutanol synthesis. Initially, slr0168, encoding a hypothetical protein, was selected as an integration site for the third copy of $k i v d^{S 286 T}$, since it has been suggested slr0168 knock-out has no effects on e.g. phenotypes and metabolisms in Synechocystis [34]. Two strains with three copies of kivd ${ }^{S 286 T}$ were generated: HX15 with a His-tagged $k i v d^{S 286 T}$ and HX28 with a Flag-tagged $k i v d^{S 286 T}$, both in the slr0168 site (Fig. 6A). As control, strain HX16 with two copies of kivd ${ }^{S 286 T}$ was generated (Fig. 6A). Unexpectedly, both strains HX15 and HX28 showed a growth lag in the beginning of cultivation, but were able to achieve a higher maximum $\mathrm{OD}_{750}$ and survived for a longer period, until day 14, when compared with control strain HX16 (Fig. 6C). Maximal isobutanol production by HX15 and HX28 were $129 \mathrm{mg} \mathrm{L}^{-1}$ and $138 \mathrm{mg} \mathrm{L}^{-1}$, $57 \%$ and $68 \%$ improvements compared to by strain HX16 (Fig. 6D). It is interesting to observe the minor but distinct production differences between HX15 and HX28, maybe as a consequence of the different tags attached in the $\mathrm{N}$ terminal of $\mathrm{Kivd}^{\mathrm{S} 286 \mathrm{~T}}$. Previously, protein engineering was successfully performed on Kivd and the strain introduced with the engineered Kivd ${ }^{\mathrm{S} 286 \mathrm{~T}}$ showed more than threefold increase in the production of isobutanol [15]. By modulating the copy numbers of kivd ${ }^{S 286 T}$ in Synechocystis cells, the total Kivd ${ }^{\mathrm{S} 26 \mathrm{~T}}$ protein expression increased, resulting a 2.7 -fold increase of isobutanol 
(A)

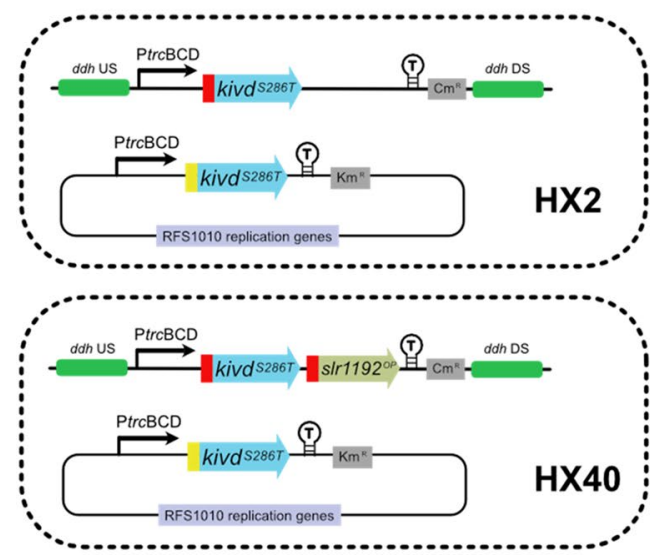

(C)

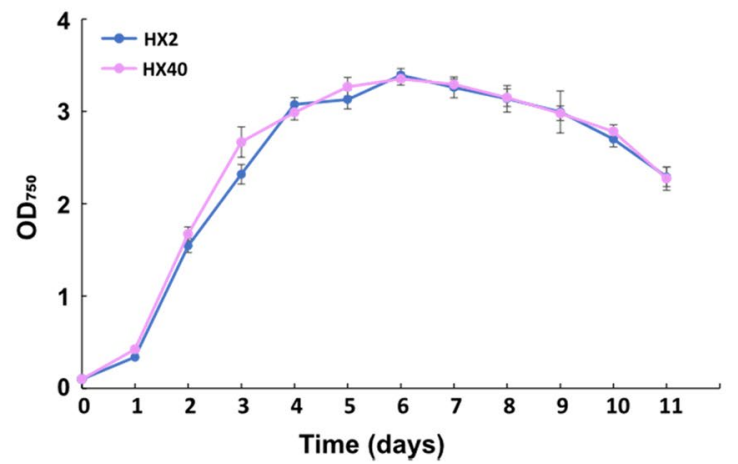

(B)

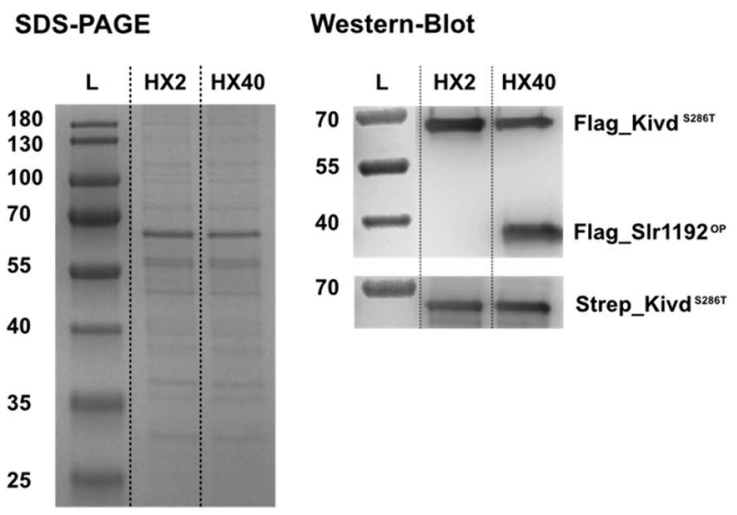

(D)

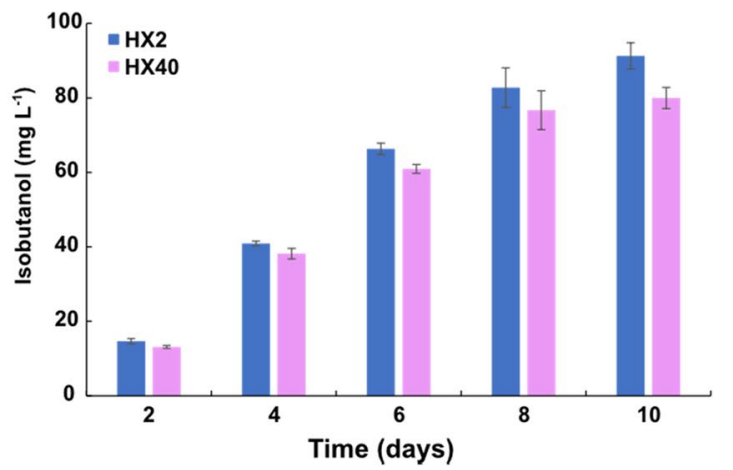

Fig. 5 Schematic overview of genetic constructs used and comparison of growth, protein levels (SDS-PAGE/Western-immunoblot) and isobutanol production in engineered Synechocystis PCC 6803 strains HX2 and HX40. A Schematic presentation of the genetic constructs in the engineered strains. kivd ${ }^{\text {S286T: }}$ : encodes a-ketoisovalerate decarboxylase (Lactococcus lactis); s/r1192 ${ }^{\mathrm{PP}}$ : encodes codon optimized alcohol dehydrogenase (Synechocystis). Kivd ${ }^{\mathrm{S} 286 \mathrm{~T}}$ expressed on self-replicating plasmids was Strep-tagged at the N-terminal; SIr $1192^{\mathrm{OP}}$ and Kivd ${ }^{\mathrm{S} 286 \mathrm{~T}}$ expressed in the $d d h$ (s/r1556) site of chromosome was Flag-tagged at the N-terminal. B SDS-PAGE (left) and Western-immunoblot (right). Each lane represents result from respective strain. L: ladder (in kDa). For SDS-PAGE, $5 \mu \mathrm{g}$ of total soluble protein were loaded for each strain. For Western-immunoblot, $5 \mu \mathrm{g}$ and $20 \mu \mathrm{g}$ of total soluble protein were loaded for each strain to detect Strep-tagged and Flag-tagged protein, respectively. C Growth curves of the engineered strains during 11-day cultivation. D Isobutanol production at day 2, 4, 6, and 8 of indicated strains. Results are the mean of three biological replicates, each with three technical replicates. Error bars represent standard deviation

production, compared to the previously best-performing isobutanol-producing strain pEEK2-ST [12].

The slr0168 site was chosen as an integrate site for introduction of the third copy of $k i v d^{S 286 T}$. The resulting strains HX15 and HX28 both showed a lag phase. To alleviate growth deficiency caused by slr0168 knockout, it is important to further screen for other suitable integration sites to introduce the third copy of $k i v d^{S 286 T}$. As shown in Fig. 1, there are several competing pathways for isobutanol biosynthesis. Therefore, via integrating the third copy of kivd ${ }^{S 286 T}$ into different sites of chromosome, the overall cellular carbon flux may be modified to favor isobutanol synthesis. $g \operatorname{lgC}$ encodes glucose-1-phosphate adenylyltransferase which catalyzes the first reaction of glycogen synthesis, a major carbohydrate storage compound in cyanobacteria. $\Delta g \operatorname{lgC}$ strain of Synechococcus showed significantly, 2.5 times, increased isobutanol production [13]. By integrating the third copy of kivd $d^{S 286 T}$ into the $g l g C$ site, strain HX47 was generated (Fig. 6A). The growth of HX47, similar to HX15, was impaired between days $0-2$ when cultivated under $50 \mu \mathrm{mol}$ photons $\mathrm{m}^{-2} \mathrm{~s}^{-1}$ (Fig. 6C), though it was previously reported that the growth retardation of $\Delta g \lg C$ mutant was only observed when cultivated under high light conditions $(>80 \mu \mathrm{mol}$ photons $\mathrm{m}^{-2} \mathrm{~s}^{-1}$ ) [35]. As for isobutanol production, HX 47 produced $144 \mathrm{mg} \mathrm{L}^{-1}$ (Fig. 6D), slightly higher than strain $\mathrm{HX} 15$. Interestingly, HX47 continued to produce isobutanol, 4-6 $\mathrm{mg} \mathrm{L}^{-1} \mathrm{OD}^{-1} \mathrm{~d}^{-1}$, for 8 days, resulting in the highest production in short-term 
(A)

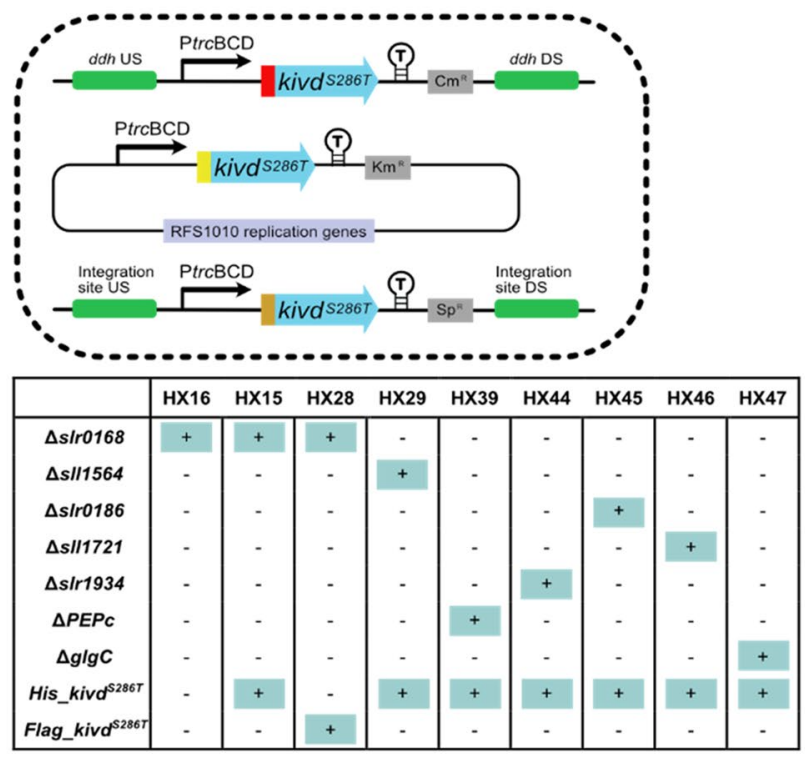

(C)

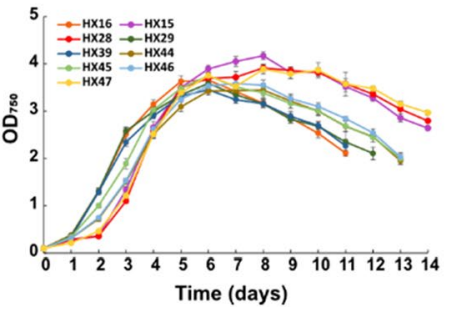

(D)

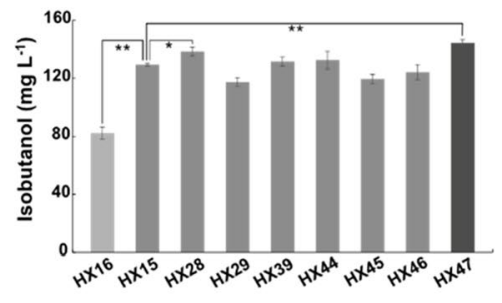

(B)

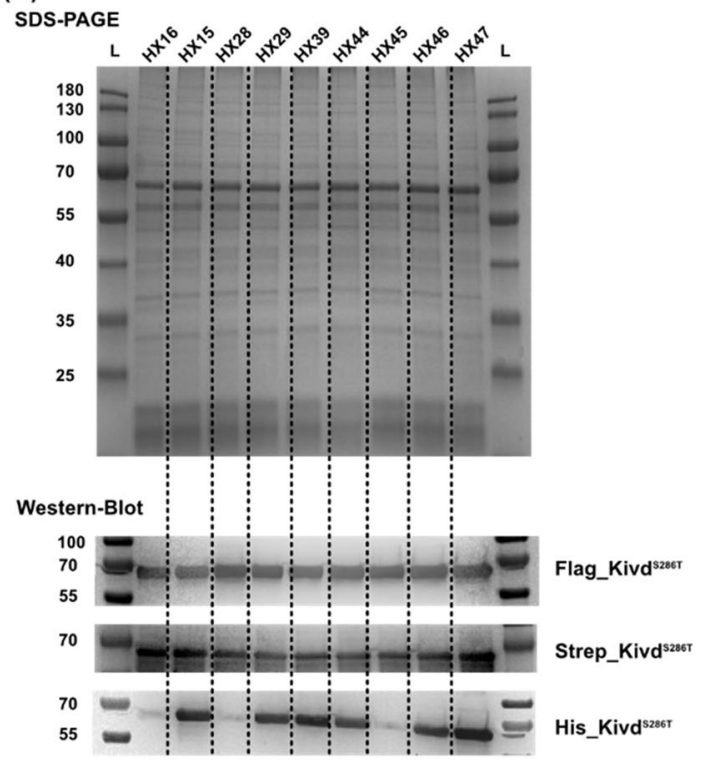

(E)

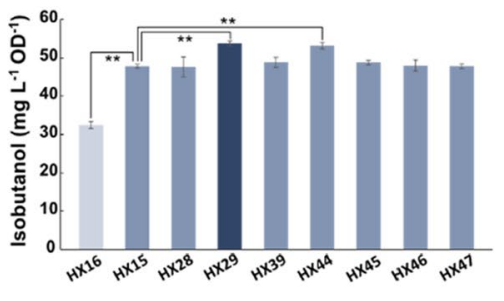

Fig. 6 Schematic overview of genetic constructs used and comparison of growth, protein levels (SDS-PAGE/Western-immunoblot) and isobutanol production/isobutanol production per cell in engineered Synechocystis PCC 6803 strains HX15-16, HX28-29, HX39, and HX44-47. A Engineered Synechocystis strains with different integration sites for the third copy of kivd ${ }^{5286 T}$. slr0168: encodes a hypothetical protein; s/11564 and s/r0186: encode 2-isopropylmalate synthase; s/11721 and s/r1934: encode pyruvate dehydrogenase; PEPC: encodes phosphoenolpyruvate carboxylase; glgC: encodes glucose-1-phosphate adenylyltransferase. HX16 was generated as a control strain. kivd ${ }^{5286 T}$ : encodes a-ketoisovalerate decarboxylase (Lactococcus lactis). Kivd ${ }^{\text {S286T }}$ expressed on self-replicating plasmids was Strep-tagged at the N-terminal; Kivd ${ }^{\text {S286T }}$ expressed in the $d d h$ (s/r1556) site of chromosome was Flag-tagged at the $\mathrm{N}$-terminal; Kivd ${ }^{\mathrm{S} 286 \mathrm{~T}}$ expressed in the third integration site of chromosome was either His-tagged or Flag-tagged at the N-terminal. B SDS-PAGE (top) and Western-immunoblot (bottom). Each lane represents result from respective strain. L: ladder (in $\mathrm{kDa}$ ). For SDS-PAGE, $5 \mu \mathrm{g}$ of total soluble protein were loaded for each strain. For Western-immunoblot, $5 \mu \mathrm{g}, 20 \mu \mathrm{g}$, and $20 \mu \mathrm{g}$ of total soluble protein were loaded for each strain to detect Strep-tagged, Flag-tagged, and His-tagged protein, respectively. C Growth curves of the engineered strains during 14-day cultivation. D Maximal isobutanol production observed of indicated strains. E Maximal isobutanol production per cell observed of indicated strains. "+" and "- "indicate with and without target gene manipulation, respectively. Results are the mean of three biological replicates, each with three technical replicates. Error bars represent standard deviation. Asterisk represents significant difference between different strains (one-way ANOVA, ${ }^{*} p<0.05,{ }^{* *} p<0.005$ )

screening experiments so far (Table 2). In contrast to what has been reported before in Synechococcus [13], no dramatic improvement in isobutanol production was observed in $\triangle g l g C$ Synechocystis strain HX47. This may be explained by the experimental conditions used including different light intensities.

Pyruvate is the precursor of the 2-keto acid pathway, a central metabolite which is further metabolized into different essential metabolites in various metabolic pathways. Thus, increasing the pyruvate pool is a relevant strategy to provide more precursor for isobutanol synthesis. In Synechocystis, pyruvate is generated from phosphoenolpyruvate (PEP), catalyzed by pyruvate kinase (PK), while phosphoenolpyruvate carboxylase (PEPc) is competing with PK for the shared substrate PEP (Fig. 1). Enhanced 2-ketoisovalerate production was observed after knocking out $P E P C$ in the bacterium Corynebacterium glutamicum [36]. Therefore, PEPc (sll0920) was chosen as an integration site to introduce the third copy of $k i v d^{S 286 T}$ to generate strain HX39 (Fig. 6A). As suspected, 
Table 2 Isobutanol production rate per cell in the engineered Synechocystis PCC 6803 strains

\begin{tabular}{|c|c|c|c|c|c|c|}
\hline \multirow{2}{*}{$\begin{array}{l}\text { Strain }\left(\mathrm{mg} \mathrm{L}^{-1}\right. \\
\left.\mathrm{OD}^{-1} \text { day }^{-1}\right)\end{array}$} & \multicolumn{6}{|l|}{ Time } \\
\hline & Day 0-1 & Day 2-3 & Day 4-5 & Day 6-7 & Day 8-9 & Day $10-11$ \\
\hline HX16 & $3.2 \pm 0.26$ & $4.2 \pm 0.20$ & $3.7 \pm 0.32$ & $2.4 \pm 0.59$ & $1.0 \pm 0.38$ & \\
\hline HX15 & $4.2 \pm 0.21$ & $3.9 \pm 0.31$ & $4.7 \pm 0.02$ & $4.6 \pm 0.29$ & $2.7 \pm 0.52$ & $1.7 \pm 0.22$ \\
\hline HX28 & $4.1 \pm 0.22$ & $4.5 \pm 0.06$ & $5.3 \pm 0.13$ & $4.1 \pm 0.27$ & $3.4 \pm 0.22$ & $2.1 \pm 0.18$ \\
\hline HX29 & $5.4 \pm 0.19$ & $6.0 \pm 0.29$ & $4.6 \pm 0.27$ & $3.9 \pm 0.11$ & $1.7 \pm 0.36$ & \\
\hline HX39 & $6.2 \pm 0.26$ & $6.6 \pm 0.13$ & $5.9 \pm 0.19$ & $3.9 \pm 0.42$ & $2.2 \pm 0.24$ & \\
\hline HX44 & $6.0 \pm 0.17$ & $6.0 \pm 0.25$ & $5.5 \pm 0.20$ & $4.9 \pm 0.10$ & $3.7 \pm+0.66$ & \\
\hline HX45 & $6.1 \pm 0.45$ & $5.3 \pm 0.20$ & $4.9 \pm 0.24$ & $3.5 \pm 0.15$ & $2.5 \pm 0.17$ & \\
\hline HX46 & $5.1 \pm 0.55$ & $5.3 \pm 0.24$ & $5.2 \pm 0.39$ & $4.8 \pm 0.37$ & $2.8 \pm 0.35$ & \\
\hline HX47 & $5.3 \pm 0.32$ & $4.8 \pm 0.20$ & $5.4 \pm 0.16$ & $4.2 \pm 0.20$ & $4.7 \pm 0.30$ & $0.9 \pm 0.17$ \\
\hline
\end{tabular}

The highest isobutanol production rate per cell of each strain is shown in bold. Results are the mean of three biological replicates, each with three technical replicates. Errors represent standard deviation

we were unable to generate a fully segregated strain, since PEPc is a key enzyme for carbon fixation in cyanobacteria with a main function to produce oxaloacetate (OAA) to feed into the Tricarboxylic acid (TCA) cycle (Fig. 1). Intermediates of the TCA cycle are used for synthesis of glutamate and aspartate family amino acids. Because of the same reason, instead of knocking out/knocking down PEPc in Synechococcus, Cheah et al. [37] heterologously introduced a revert reaction catalyzed by the enzyme PEP carboxykinase (PCK) from E. coli to convert OAA into PEP. Strain HX39 grew as fast as control strain HX16 between days 0 and 4 , reaching slightly lower $\mathrm{OD}_{750}$ than HX16 (Fig. 6C). After 10-day cultivation, the final isobutanol production was $132 \mathrm{mg} \mathrm{L}^{-1}$ (Fig. 6D), similar to in HX15. Interestingly, among all the engineered strains, strain HX39 showed the highest production rate per cell (6.6 $\mathrm{mg} \mathrm{L}^{-1} \mathrm{OD}^{-1} \mathrm{~d}^{-1}$ ) between days 2 and 3 (Table 2 ).

Apart from increasing PEP availability for pyruvate synthesis, another approach to increase pyruvate pool is to decrease the enzymatic reactions using pyruvate as substrate. One important enzymatic reaction using pyruvate as substrate is catalyzed by pyruvate dehydrogenase complex for acetyl-CoA synthesis (Fig. 1). In Synechocystis, the pyruvate dehydrogenase complex consists of three enzymes: pyruvate dehydrogenase (encoded by sll1721 and slr1934), dihydrolipoamide acetyltransferase (encoded by sll1841), and dihydrolipoamide dehydrogenase (encoded by slr1096). Since pyruvate dehydrogenase is regarded as a rate-limiting step for this reaction, both sll1721 and slr1934 were assigned as integration sites for the introduction of the third copy of kivd ${ }^{S 286 T}$ to generate strains HX46 and HX44 (Fig. 6A). We were unable to get fully segregated cells for both strains, as pyruvate dehydrogenase complex catalyzes the main route for acetylCoA synthesis, which is further involved in TCA cycle and used for e.g. lipid synthesis. Even though the growth rates of HX44 and HX46 between days 0 and 4 were slower than control strain HX16 and faster than strain HX15 (Fig. 6C), both strains produced similar isobutanol as HX15, $132 \mathrm{mg} \mathrm{L}^{-1}$ and $124 \mathrm{mg} \mathrm{L}^{-1}$, respectively (Fig. 6D). However, after normalizing isobutanol production to per cell, strain $\mathrm{HX} 44$ reached $53 \mathrm{mg} \mathrm{L}^{-1} \mathrm{OD}^{-1}$, which is significantly higher than control strain HX15 (Fig. 6E).

As presented previously, engineered Synechocystis cells produced two end-products, isobutanol and 3M1B, after heterologous expression of kivd from L. lactis [14]. Based on that, protein engineering was performed on Kivd, resulting in improved isobutanol production as well as an improved isobutanol-to-3M1B molar ratio [15]. To further shift the carbon flow from $3 \mathrm{M} 1 \mathrm{~B}$ to isobutanol synthesis, leuA, encoding 2-isopropylmalate synthase, was selected as a target for the third copy of kivd ${ }^{S 286 T}$ integration (Fig. 1). Limited information is available for 2-isopropylmalate synthase of Synechocystis, however, two genes, slr0186 and sll1564, are annotated as leuA. Thus, two strains were generated, HX45 and HX29, through the integration of the third copy of kivd $d^{S 286 T}$ in the slr0186 site and sll1564 site (Fig. 6A), respectively. Interestingly, strain HX29 got fully segregated, but not strain HX45. Furthermore, a higher isobutanol-to-3M1B molar ratio was observed for strain HX45, when compared to strain HX15 (Additional file 1: Fig. S3). Both facts indicate that slr0186 encoding LeuA plays a major role in leucine synthesis as well as 3M1B synthesis. For sll1564 encoding LeuA, apart from LeuA activity, it is also annotated as citramalate synthase. Thus, complete knock-out of slr0186 is lethal for Synechocystis cells. Consistently, HX29 grew as fast as control strain HX16, while HX45 showed slower growth between days 0 and 4 . After 12-day cultivation, isobutanol production per cell of $\mathrm{HX} 45$ reached $49 \mathrm{mg} \mathrm{L}^{-1} \mathrm{OD}^{-1}$ (Fig. 6E), and HX29 reached $54 \mathrm{mg} \mathrm{L}^{-1}$ 
$\mathrm{OD}^{-1}$, the highest isobutanol production per cell of all engineered strains (Fig. 6E).

Multiple integration sites have been experimentally examined for effects on isobutanol production. By knockout/knock-down of the discussed genes encoding key enzymes of potential competing pathways, more carbon flux is expected to be directed towards isobutanol synthesis. The highest isobutanol production, $144 \mathrm{mg} \mathrm{L}^{-1}$, was obtained by strain HX47 (Fig. 6D), 76\% improvement compared to control strain HX16. Strain HX29 showed the highest isobutanol production per cell, $54 \mathrm{mg} \mathrm{L}^{-1}$ $\mathrm{OD}^{-1}$ (Fig. 6E), 69\% improvement compared to control strain HX16.

Some strains were not fully segregated, meaning instead of knocked out, the selected competing pathway was knocked down. When comparing the His-tagged Kivd $^{\text {S286T }}$ expression levels of all engineered strains, similar expression level was observed between strains fully segregated and strain not fully segregated (Fig. 6B). Interestingly, HX47, a strain not fully segregated even had more His-tagged Kivd ${ }^{\mathrm{S} 286 \mathrm{~T}}$ expressed than other strains (Fig. 6B; Additional file 1: Table S4). From these results, it may be inferred that in strains without fully segregation, the majority of the chromosomes already contain $k i v d^{S 286 T}$ in the assigned integration sites. Furthermore, when a protein-coding gene $k i v d^{S 286 T}$ was introduced into different integration sites of the chromosome, the resulting protein expression levels were not consistent (Fig. 6B), a result from different genomic context with regulations in both transcription and translation levels [38]. On the other hand, when knocking out essential genes with a traditional homologous double recombination method, it is sometimes difficult to get fully segregated strains. Alternatively, antisense RNA [37] may be employed as a tool for downregulation of those essential genes to further optimize isobutanol production in Synechocystis.

Compared to strain HX15, the newly generated engineered strain HX47 showed significantly increased isobutanol production whereas strains HX29 and HX44 showed significantly increased isobutanol production per cell (Fig. 6D, E). Three hypotheses can be made: first, there is no obvious effect when knocking out/knocking down selected competing pathways singly, providing a new investigation direction to knock out/knock down all of them simultaneously to effectively concentrate carbon flow into the 2-keto acid pathway for isobutanol synthesis. As an approach for simultaneous repression of multiple genes, clustered regularly interspaced short palindromic repeats interference (CRISPRi) has been developed for Synechocystis [39], and was successfully used to improve fatty alcohol production in Synechocystis [40]; Second, knock-out/knock-down of competing pathways is effective for enhancement of precursor availability for isobutanol synthesis, however, Kivd ${ }^{\mathrm{S} 286 \mathrm{~T}}$ is still a bottleneck for isobutanol synthesis in Synechocystis cells. Thus, to resolve the bottleneck $\mathrm{Kivd}^{\mathrm{S} 286 \mathrm{~T}}$, two approaches may be adopted: one is to further improve its catalytic activity by protein engineering based on structure analysis as well as computational simulation; another one is to develop new strategies for further improvement of $\mathrm{Kivd}^{\mathrm{S} 286 \mathrm{~T}}$ protein amount in Synechocystis cells; Third, after introducing three copies of kivd ${ }^{S 286 T}$ in Synechocystis cells, enough $\mathrm{Kivd}^{\mathrm{S} 286 \mathrm{~T}}$ are ready to convert 2-ketoisovalerate into isobutyraldehyde. Therefore, Kivd ${ }^{\mathrm{S} 286 \mathrm{~T}}$ is not the rate-limiting enzyme for isobutanol synthesis. Instead, in this stage, there is (are) other bottleneck(s) in isobutanol synthesis pathway, and further investigation are needed to precisely find out the potential bottleneck(s). Regarding the last two hypotheses, only after successfully removing bottleneck(s), the contribution of competing pathway knock-out/knock-down could be manifested from the resulting isobutanol production in engineered Synechocystis cells.

\section{Conclusion}

In summary, this is the first demonstration of the feasibility to generate an engineered Synechocystis strain with a complete 2-keto acid pathway integrated. In short-term screening experiments, the resulting strain HX42 was able to produce $98 \mathrm{mg}$ isobutanol $\mathrm{L}^{-1}$, an $88 \%$ increase compared to the previously reported bestproducing strain pEEK2-ST (Fig. 7) [12]. By fine-tuning expression of the key enzyme Kivd ${ }^{\mathrm{S} 286 \mathrm{~T}}$ from both transcription and translation levels, the resulting strain HX2 and HX15 showed 70\% and 148\% increase of isobutanol production compared with strain pEEK2-ST

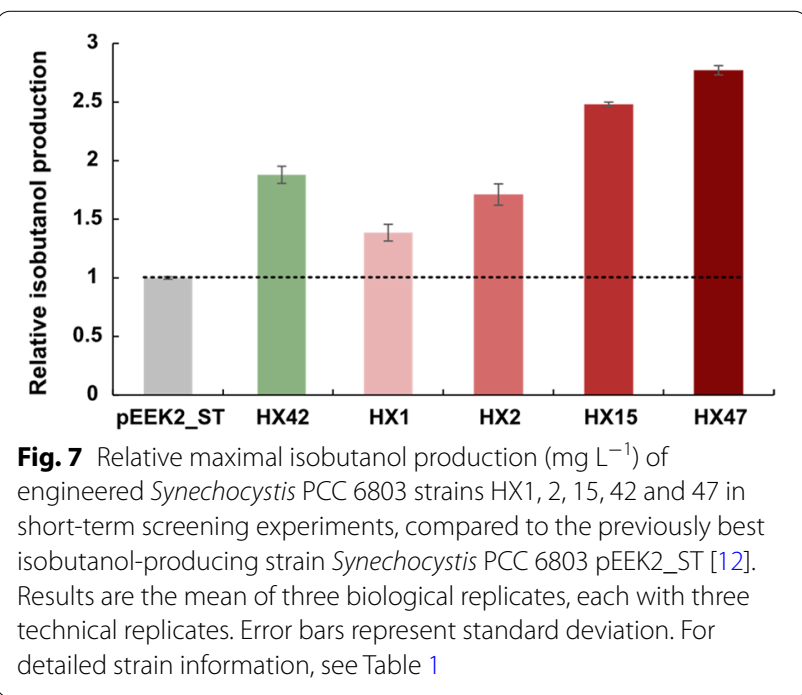


(Fig. 7). Further improved isobutanol production, $144 \mathrm{mg} \mathrm{L}^{-1}$, was obtained by interrupting the glycogen biosynthesis pathway (strain HX47) (Fig. 7). The present results suggest that a combination of integrating a complete 2-keto acid pathway and meanwhile keeping high protein expression of $\mathrm{Kivd}^{\mathrm{S} 286 \mathrm{~T}}$ is a potential strategy to further improve isobutanol production in Synechocystis. Moreover, further simultaneous repression of multiple competing pathways of isobutanol synthesis pathway is likely to concentrate more carbon flux into the 2-keto acid pathway for isobutanol synthesis. The final strain HX47 was able to reach much higher isobutanol production and production rate if cultivated in a condition with precisely controlled $\mathrm{pH}$, as detailed before [12]. Finally, in order to successfully use Synechocystis as a novel platform for production of photosynthetic isobutanol, one key factor is to develop a chemostat system suitable for cyanobacteria cultivation, combined with an automatic isobutanol harvesting and upgrade system.

\begin{abstract}
Abbreviations
3M1B: 3-Methyl-1-butanol; Adh: Alcohol dehydrogenase; AlsS: Acetolactate synthase; $\mathrm{BCD}$ : Bicistronic design; $\mathrm{Cm}^{\mathrm{R}}$ : Chloramphenicol resistance; CRISPRi: Clustered regularly interspaced short palindromic repeats interference; IlvC: Acetohydroxy-acid isomeroreductase; llvD: Dihydroxy-acid dehydrase; Kivd: a-Ketoisovalerate decarboxylase; NSI: Neutral site I; OAA: Oxaloacetate; $\mathrm{OD}_{750}$ : Optical density; PCK: PEP carboxykinase; PEP: Phosphoenolpyruvate; PEPC: Phosphoenolpyruvate carboxylase; PK: Pyruvate kinase; RBSs: Ribosome binding sites; RiboJ: Self-cleaving ribozyme; Rubisco: Ribulose 1,5-bisphosphate carboxylase/oxygenase; Slr1192 ${ }^{\mathrm{OP}}$ : Codon optimized version of SIr1192; Sp $p^{R}$ : Spectinomycin resistance; Synechococcus: Synechococcus elongatus PCC 7942; Synechocystis: Synechocystis PCC 6803; TCA cycle: Tricarboxylic acid cycle.
\end{abstract}

\section{Supplementary Information}

The online version contains supplementary material available at https://doi. org/10.1186/s12934-022-01738-z.

Additional file 1. Fig. S1: Schematic overview of genetic constructs used and Western-immunoblot results of engineered Synechocystis PCC 6803 strains HXO and HX6. (A) Schematic presentation of the genetic constructs in the engineered strains. kivd ${ }^{5286 T}$ : encodes a-ketoisovalerate decarboxylase (Lactococcus lactis). alsS: encodes acetolactate synthase (Bacillus subtilis). Kivd ${ }^{5286 T}$ expressed on self-replicating vectors was Strep-tagged at the $\mathrm{N}$-terminal; AlsS expressed in the ddh (s/r1556) site of chromosome was His-tagged at the N-terminal. (B) Western-immunoblot results of strains $\mathrm{HXO}$ and HX6. Each lane represents result from respective strain. $5 \mu \mathrm{g}$ and $20 \mathrm{\mu g}$ of total soluble protein were loaded for each strain to detect Streptagged Kivd ${ }^{\text {S286T }}$ and His-tagged AlsS, respectively. Fig. S2: Comparison of growth in engineered Synechocystis PCC 6803 strains HX0, HX5, HX7, HX8, and HX9 during 8-day cultivation. Results are the mean of three biological replicates, each with three technical replicates. Error bars represent standard deviation. Fig. S3: Schematic overview of genetic constructs used and comparison of molar ratio of isobutanol and 3-methyl-1-butanol (3M1B) of engineered Synechocystis PCC 6803 strains HX15, HX29, and HX45. (A) Molar ratio of isobutanol and $3 \mathrm{M} 1 \mathrm{~B}$ of indicated strains, calculated based on the isobutanol production measured on day 4. (B) Schematic presentation of the genetic constructs in the engineered strains. Asterisk represents significant difference between HX45 and HX15 (One-way ANOVA, $\mathrm{p}$ $<0.05)$. Results are the mean of three biological replicates, each with three technical replicates. Error bars represent standard deviation. Table S1: Sequences of codon optimized synthetic genes used in this study.

Table S2: Plasmids used in this study. Expressed genes in bold. Table S3: Oligonucleotides used in this study. Table S4: Expression quantification of heterologously expressed enzymes. The expression level of each protein is presented by the corresponding band intensity. The unit is intensity $x \mathrm{~mm}$

\section{Authors' contributions}

$H X$ and PL designed the project. HX performed all the experiments, analyzed the data and wrote the manuscript. PL supervised the work and revised the manuscript. Both authors read and approved the final manuscript.

\section{Funding}

Open access funding provided by Uppsala University. This study was funded by Energimyndigheten and NordForsk with Grant numbers P46607-1 (CyanoFuels) and 82845 (NordAqua).

\section{Availability of data and materials}

The datasets used during the current study are available from the corresponding author on reasonable request.

\section{Declarations}

Ethics approval and consent to participate

Not applicable in this section.

\section{Consent for publication}

Not applicable.

\section{Competing interests}

The authors declare that they have no competing interests.

Received: 18 October 2021 Accepted: 3 January 2022 Published: 1 February 2022

\section{References}

1. Zhou YJ, Kerkhoven EJ, Nielsen J. Barriers and opportunities in bio-based production of hydrocarbons. Nat Energy. 2018;3(11):925-35.

2. Atsumi S, Hanai T, Liao JC. Non-fermentative pathways for synthesis of branched-chain higher alcohols as biofuels. Nature. 2008;451(7174):86-9.

3. Fu C, Li Z, Jia C, Zhang W, Zhang Y, Yi C, et al. Recent advances on biobased isobutanol separation. Energy Convers Manage X. 2021;10:100059.

4. Liu X, Xie H, Roussou S, Lindblad P. Current advances in engineering cyanobacteria and their applications for photosynthetic butanol production. Curr Opin Biotechnol. 2021;73:143-50.

5. Liu X, Xie H, Roussou S, Miao R, Lindblad P. Engineering cyanobacteria for photosynthetic butanol production. In: Rögner M, editor. Photosynthesis: biotechnological applications with micro-algae. Berlin: Walter de Gruyter $\mathrm{GmbH} ; 2021$.

6. Lee WH, Seo SO, Bae YH, Nan H, Jin YS, Seo JH. Isobutanol production in engineered Saccharomyces cerevisiae by overexpression of 2-ketoisovalerate decarboxylase and valine biosynthetic enzymes. Bioprocess Biosyst Eng. 2012;35(9):1467-75.

7. Hasegawa S, Jojima T, Suda M, Inui M. Isobutanol production in Corynebacterium glutamicum: suppressed succinate by-production by pckA inactivation and enhanced productivity via the Entner-Doudoroff pathway. Metab Eng. 2020;59:24-35.

8. Lu J, Brigham CJ, Gai CS, Sinskey AJ. Studies on the production of branched-chain alcohols in engineered Ralstonia eutropha. Appl Microbiol Biotechnol. 2012;96(1):283-97.

9. Higashide W, Li Y, Yang Y, Liao JC. Metabolic engineering of Clostridium cellulolyticum for production of isobutanol from cellulose. Appl Environ Microbiol. 2011;77(8):2727-33.

10. Li S, Wen J, Jia X. Engineering Bacillus subtilis for isobutanol production by heterologous Ehrlich pathway construction and the biosynthetic 
2-ketoisovalerate precursor pathway overexpression. Appl Microbiol Biotechnol. 2011;91(3):577-89.

11. Atsumi S, Higashide W, Liao JC. Direct photosynthetic recycling of carbon dioxide to isobutyraldehyde. Nat Biotechnol. 2009;27(12):1177-80.

12. Miao R, Xie H, Lindblad P. Enhancement of photosynthetic isobutanol production in engineered cells of Synechocystis PCC 6803. Biotechnol Biofuels. 2018;11:267.

13. Li X, Shen CR, Liao JC. Isobutanol production as an alternative metabolic sink to rescue the growth deficiency of the glycogen mutant of Synechococcus elongatus PCC 7942. Photosynth Res. 2014;120(3):301-10.

14. Miao R, Liu X, Englund E, Lindberg P, Lindblad P. Isobutanol production in Synechocystis PCC 6803 using heterologous and endogenous alcohol dehydrogenases. Metab Eng Commun. 2017;5:45-53.

15. Miao R, Xie H, Ho FM, Lindblad P. Protein engineering of alpha-ketoisovalerate decarboxylase for improved isobutanol production in Synechocystis PCC 6803. Metab Eng. 2018:47:42-8.

16. Rippka R, Deruelles J, Waterbury JB, Herdman M, Stanier RY. Generic assignments, strain histories and properties of pure cultures of cyanobacteria. Microbiology. 1979;111(1):61

17. Englund E, Andersen-Ranberg J, Miao R, Hamberger B, Lindberg P. Metabolic engineering of Synechocystis sp. PCC 6803 for production of the plant diterpenoid manoyl oxide. ACS Synth Biol. 2015;4(12):1270-8.

18. Englund E, Shabestary K, Hudson EP, Lindberg P. Systematic overexpression study to find target enzymes enhancing production of terpenes in Synechocystis PCC 6803, using isoprene as a model compound. Metab Eng. 2018;49:164-77.

19. Tamagnini P, Troshina O, Oxelfelt F, Salema R, Lindblad P. Hydrogenases in Nostoc sp. strain PCC 73102, a strain lacking a bidirectional enzyme. Appl Environ Microbiol. 1997;63(5):1801-7.

20. Atsumi S, Li Z, Liao JC. Acetolactate synthase from Bacillus subtilis serves as a 2-ketoisovalerate decarboxylase for isobutanol biosynthesis in Escherichia coli. Appl Environ Microbiol. 2009;75(19):6306-11.

21. Mustila H, Kugler A, Stensjö K. Isobutene production in Synechocystis sp. PCC 6803 by introducing alpha-ketoisocaproate dioxygenase from Rattus norvegicus. Metab Eng Commun. 2021;12:e00163.

22. Rodrigues JS, Lindberg P. Metabolic engineering of Synechocystis sp. PCC 6803 for improved bisabolene production. Metab Eng Commun. 2021:12:e00159.

23. Liu X, Miao R, Lindberg P, Lindblad P. Modular engineering for efficient photosynthetic biosynthesis of 1-butanol from $\mathrm{CO}_{2}$ in cyanobacteria. Energy Environ Sci. 2019;12(9):2765-77.

24. Gao Z, Zhao H, Li Z Tan X, Lu X. Photosynthetic production of ethanol from carbon dioxide in genetically engineered cyanobacteria. Energy Environ Sci. 2012;5(12):9857-65.

25. Formighieri C, Melis A. A phycocyanin.phellandrene synthase fusion enhances recombinant protein expression and beta-phellandrene (monoterpene) hydrocarbons production in Synechocystis (cyanobacteria). Metab Eng. 2015;32:116-24

26. Armshaw P, Carey D, Sheahan C, Pembroke JT. Utilising the native plasmid, pCA2.4, from the cyanobacterium Synechocystis sp. strain PCC6803 as a cloning site for enhanced product production. Biotechnol Biofuels. 2015;8:201.

27. Choi SY, Wang JY, Kwak HS, Lee SM, Um Y, Kim Y, et al. Improvement of squalene production from $\mathrm{CO}_{2}$ in Synechococcus elongatus PCC 7942 by metabolic engineering and scalable production in a photobioreactor. ACS Synth Biol. 2017;6(7):1289-95.

28. Lindberg P, Park S, Melis A. Engineering a platform for photosynthetic isoprene production in cyanobacteria, using Synechocystis as the model organism. Metab Eng. 2010;12(1):70-9.

29. Ng WO, Zentella R, Wang Y, Taylor JS, Pakrasi HB. PhrA, the major photoreactivating factor in the cyanobacterium Synechocystis sp. strain PCC 6803 codes for a cyclobutane-pyrimidine-dimer-specific DNA photolyase. Arch Microbiol. 2000;173(5-6):412-7.

30. Labarre J, Chauvat F, Thuriaux P. Insertional mutagenesis by random cloning of antibiotic resistance genes into the genome of the cyanobacterium Synechocystis strain PCC 6803. J Bacteriol. 1989:171(6):3449-57.

31. Mutalik VK, Guimaraes JC, Cambray G, Lam C, Christoffersen MJ, Mai QA, et al. Precise and reliable gene expression via standard transcription and translation initiation elements. Nat Methods. 2013;10(4):354-60.
32. Lou C, Stanton B, Chen YJ, Munsky B, Voigt CA. Ribozyme-based insulator parts buffer synthetic circuits from genetic context. Nat Biotechnol. 2012:30(11):1137-42.

33. Terai G, Kamegai S, Taneda A, Asai K. Evolutionary design of multiple genes encoding the same protein. Bioinformatics. 2017;33(11):1613-20.

34. Kunert A, Hagemann M, Erdmann N. Construction of promoter probe vectors for Synechocystis sp. PCC 6803 using the light-emitting reporter systems Gfp and LuxAB. J Microbiol Methods. 2000;41(3):185-94.

35. Miao X, Wu Q, Wu G, Zhao N. Changes in photosynthesis and pigmentation in an agp deletion mutant of the cyanobacterium Synechocystis sp. Biotechnol Lett. 2003;25(5):391-6.

36. Buchholz J, Schwentner A, Brunnenkan B, Gabris C, Grimm S, Gerstmeir $R$, et al. Platform engineering of Corynebacterium glutamicum with reduced pyruvate dehydrogenase complex activity for improved production of L-lysine, L-valine, and 2-ketoisovalerate. Appl Environ Microbiol. 2013;79(18):5566-75.

37. Cheah YE, XU Y, Sacco SA, Babele PK, Zheng AO, Johnson CH, et al. Systematic identification and elimination of flux bottlenecks in the aldehyde production pathway of Synechococcus elongatus PCC 7942. Metab Eng. 2020;60:56-65.

38. Atkinson TJ, Halfon MS. Regulation of gene expression in the genomic context. Comput Struct Biotechnol J. 2014;9:e201401001.

39. Yao L, Cengic I, Anfelt J, Hudson EP. Multiple gene repression in cyanobacteria using CRISPRi. ACS Synth Biol. 2016;5(3):207-12.

40. Kaczmarzyk D, Cengic I, Yao L, Hudson EP. Diversion of the long-chain acyl-ACP pool in Synechocystis to fatty alcohols through CRISPRi repression of the essential phosphate acyltransferase PIsX. Metab Eng. 2018;45:59-66

\section{Publisher's Note}

Springer Nature remains neutral with regard to jurisdictional claims in published maps and institutional affiliations.

Ready to submit your research? Choose BMC and benefit from:

- fast, convenient online submission

- thorough peer review by experienced researchers in your field

- rapid publication on acceptance

- support for research data, including large and complex data types

- gold Open Access which fosters wider collaboration and increased citations

- maximum visibility for your research: over 100M website views per year

At BMC, research is always in progress.

Learn more biomedcentral.com/submissions 